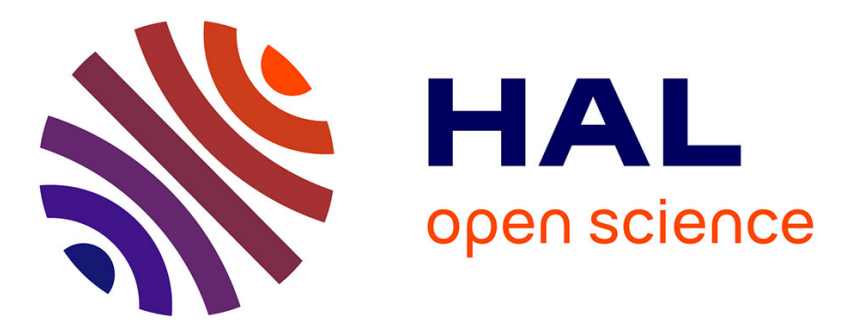

\title{
On the universality of the flow properties of soft-particle glasses
}

\author{
Tianfei Liu, Fardin Khabaz, Roger T. Bonnecaze, Michel Cloitre
}

\section{To cite this version:}

Tianfei Liu, Fardin Khabaz, Roger T. Bonnecaze, Michel Cloitre. On the universality of the flow properties of soft-particle glasses. Soft Matter, 2018, 14 (34), pp.7064-7074. 10.1039/C8SM01153B . hal-02406345

\section{HAL Id: hal-02406345 \\ https://hal.science/hal-02406345}

Submitted on 27 May 2021

HAL is a multi-disciplinary open access archive for the deposit and dissemination of scientific research documents, whether they are published or not. The documents may come from teaching and research institutions in France or abroad, or from public or private research centers.
L'archive ouverte pluridisciplinaire HAL, est destinée au dépôt et à la diffusion de documents scientifiques de niveau recherche, publiés ou non, émanant des établissements d'enseignement et de recherche français ou étrangers, des laboratoires publics ou privés. 


\title{
On the Universality of the Flow Properties of Soft-Particle Glasses
}

\author{
Tianfei Liu, ${ }^{\mathrm{a}}$ Fardin Khabaz, ${ }^{\mathrm{a}}$ Roger T. Bonnecaze ${ }^{\mathrm{a}^{*}}$ and Michel Cloitre ${ }^{\mathrm{b} \dagger}$ \\ ${ }^{a}$ McKetta Department of Chemical Engineering and Texas Materials Institute, \\ University of Texas at Austin, Austin, TX 78712 USA

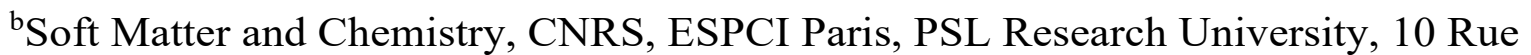 \\ Vauquelin, 75005 Paris, France
}

\begin{abstract}
We identify the minimal interparticle interactions necessary for a particle dynamics simulation to predict the structure and flow behaviour of soft particle glasses (SPGs). Generally, two kinds of forces between the particles must be accounted for in simulations of SPGs: viscous or frictional drag forces and elastic contact forces. Far field drag forces are required to dissipate energy in the simulations and capture the effect of the rheology of the suspending fluid. Elastic forces are found to be dominant compared to near-field drag or other forms of friction forces and are the most important component to compute the rheology. The shear stress, the first and second normal stress differences for different interparticle force laws collapse onto universal master curves of the Herschel-Bulkley form by non-dimensionalizing the stress with the yield stress and the shear rate with the viscosity of the suspending fluid divided by the low-frequency shear modulus. The Herschel-Bulkley exponents are close to 0.5 with a slight dependence on the repulsive pairwise elastic forces.
\end{abstract}

\footnotetext{
${ }^{*}$ rtb@che.utexas.edu

+ michel.cloitre@espci.fr
} 


\section{Introduction}

Soft particles glasses (SPGs) are composed of deformable particles that are jammed at volume fractions beyond close-packing. They cover a wide range of materials such as microgels, emulsions, block copolymer micelles, and star polymers. ${ }^{1}$ They show elastic solid behavior at rest and flow under stresses larger than the yield stress. These characteristics make them useful for coating applications, paints, pastes, textured foods, and cosmetic products. ${ }^{2}$ Similar to hard sphere glasses, soft particles glasses demonstrate nonergodicity and caged dynamics. ${ }^{3}$ However, while hard sphere glasses only experience forces due to excluded volume interactions, soft particle glasses are compressed via a bulk osmotic force and interact through an elastic repulsive potential. The phase diagram of soft particle suspensions shows strong similarities with that of hard spheres. ${ }^{3}$ Many systems like emulsions or microgels exhibit the same sequence of fluid, entropic glass, and soft glass phases upon increasing the density. ${ }^{4,5}$ For relatively monodisperse suspensions, jamming takes place at a volume fraction of about 0.64 . Above the jamming point, thermal or Brownian forces become negligible compared to contact forces, which determine the microstructure and macroscopic properties.

The shear stress $\sigma$ of soft particle glasses is well represented by the HerschelBulkley equation ${ }^{6}$ :

$$
\sigma=\sigma_{y}+k \dot{\gamma}^{m}
$$

where $\sigma_{\mathrm{y}}$ is the yield stress, $k$ the consistency, and $m$ the so-called HerschelBulkley exponent. For a long time this equation has been considered as a purely empirical, albeit convenient, tool for characterizing the nonlinear rheology of yield stress materials. More recently the problem has stimulated a lot of research with the objective to connect the microscopic properties and the macroscopic rheology of soft 
particle glasses. ${ }^{1,3,6}$ Important questions concern the origin and the prediction of the yield stress, the values of the parameters involved in the Herschel-Bulkley equation and in particular the exponent $m$. Careful experiments on well-characterized SPGs have demonstrated that the Herschel-Bulkley equation provides a good description of the flow properties of SPGs with an exponent close to $0.5 .^{4,7-13}$. In addition the flow curves measured for different particle densities have been found to collapse onto master curves when the stress and the shear rates are rescaled by appropriate parameters. ${ }^{4,7,10-13}$ These experimental findings have stimulated an intense theoretical activity resulting in a variety of descriptions based on scaling methods, ${ }^{14}$ STZ theory, ${ }^{15}$ phenomenological models like the SGR model ${ }^{16}$, and elastoplastic models and its many variants. ${ }^{17-23}$ However many features of the flow properties of soft particle glasses remain poorly understood.

Microscopic models have also been developed in order to describe and capture the macroscopic flow behavior of jammed suspensions in relation with their particle scale behavior. ${ }^{24-31}$ In $2 \mathrm{D}$ the particles are represented by circular disks which, when overlapping and only then, interact via repulsive forces. The repulsive force between two particles $\alpha$ and $\beta$ depends on the overlap distance $h_{\alpha \beta}=R_{\alpha}+R_{\beta}-r_{\alpha \beta}$, where $R_{\alpha}$ and $R_{\beta}$ are the radii of the particles and $r_{\alpha \beta}$ is their center-to-center distance. The second key ingredient is the viscous dissipation force, which in general is assumed to be the sum of the drag forces exerted on each particle by its neighbors. In general an external force is applied to keep the suspension moving and provide the applied shear strain and strain rate. Inertia is generally neglected or made negligible resulting in strongly overdamped motion. In his pioneering work, Durian took an elastic repulsive force derived from a harmonic potential and for simplicity computed the dissipation with respect to the imposed average linear shear velocity flow. Inertia was neglected. 
The resulting flow curves were found to obey the Bingham equation ( $m=1$ in Eq. 1$)$. Later on Langlois et al. allowed the particles to move independently so that the drag force between two particles was computed in proportion to their relative difference and not with respect to the background fluid velocity. ${ }^{27} \mathrm{~A}$ mass was attributed to particles but it was small enough to make inertia negligible. The flow curves were found to obey the Herschel-Bulkley equation with an exponent $m$ close to 0.5 . Different authors have implemented the soft-disk model with harmonic interactions and drawn similar conclusions. ${ }^{29,30}$ Tighe et al. identified four different shear rate dependent regimes, namely yield stress, transition, critical and viscous regimes. ${ }^{28}$ In the critical regime, the flow curve was characterized by a Herschel-Bulkley exponent of 0.5 whereas, in the viscous regime at very high shear rates, it followed the Bingham equation. The exponent $m \cong 0.5$ has also been observed in molecular dynamic simulations of 2D athermal Lenard-Jones glasses. ${ }^{20,21,26,32}$

These models are 2D in essence, which raises the question of whether they are representative of experiments. Actually, only a few 3D simulation schemes have been developed to investigate the flow of jammed materials. ${ }^{11,33,34}$ It is not yet clear if and how the microscopic ingredients of the models influence the macroscopic rheology and whether the results which have been obtained are general. The main questions concern the form of the repulsive forces acting on the particles and the nature of the viscous drag forces that controls the dissipation. In this paper we address these issues using a $3 \mathrm{D}$ micromechanical model that we have shown to successfully describe the linear and nonlinear rheology of jammed SPGs, and produce shear stress and normal stresses predictions in quantitative agreement with steady and oscillatory experiments. ${ }^{11,35} \mathrm{We}$ implement different repulsive forces corresponding to varying softness and explore the role of the dissipative drag force between particles. We show 
that the non-linear rheological properties depend on the expressions of the viscous and elastic forces but that the shear stress and normal stresses difference can be rescaled on similar universal Herschel-Bulkley master curves once the elastic modulus is used as the control parameter.

\section{Computational method}

\subsection{Model description and equations of motion}

The suspension consists of particles with a Young modulus $E$, dispersed in a solvent of viscosity $\eta_{\mathrm{s}}$, at a volume fraction $\phi$. As depicted in Fig.a, the jammed suspension flows in the $x$-direction and is subject to a velocity gradient $\dot{\gamma}$ in the $x-y$ plane. The motion of each particle is determined by the sum of the pairwise forces exerted by the neighboring particles, which can be categorized into dissipative drag forces $\mathbf{f}_{\alpha}^{\text {drag }}$ and interparticle repulsive forces associated with elastic interactions $\mathbf{f}_{\alpha \beta}^{\text {elas }}$. The total drag forces are split into two contributions, namely the far-field drag force $\mathbf{f}_{\alpha \beta}^{\mathrm{far}-\mathrm{drag}}$ and the near-field drag force $\mathbf{f}_{\alpha \beta}^{\text {near-drag }}$.

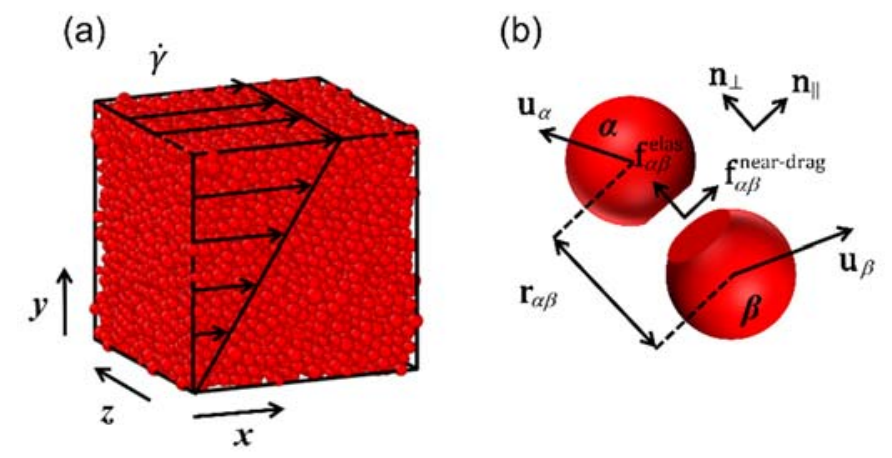

(b)

Fig. 1 Schematic representation of a sheared suspension (a) and pairwise interaction (b).

\subsubsection{Elastic forces $\mathbf{f}_{\alpha \beta}^{\text {elas }}$}

Elastic forces are the normal forces acting on the contacting facets between a particle and their neighbors. They are responsible for the mutual repulsion between two neighboring particles In the original version of the model, ${ }^{11}$ the repulsive elastic 
force has the form of a modified Hertzian force, which is known to well represent the repulsive forces between two elastomeric particles: ${ }^{36}$

$$
\mathbf{f}_{\alpha \beta}^{\text {elas }}=\frac{4}{3} C E^{*} \varepsilon_{\alpha \beta}^{n} R_{c}^{2} \mathbf{n}_{\perp}
$$

where $E^{*}=E / 2\left(1-v^{2}\right)$ is the contact modulus based on the Young modulus $E$ and Poisson ratio $v, R_{c}=R_{\alpha} R_{\beta} /\left(R_{\alpha}+R_{\beta}\right)$ is the contact radius, $\varepsilon_{\alpha \beta}=\left(R_{\alpha}+R_{\beta}-r_{\alpha \beta}\right) / R_{c}$ is the dimensionless overlap distance characterizing the degree of compression, and $\mathbf{n}_{\perp}$ is the unit vector perpendicular to the flat surface representing the particle-particle contact. The values of $C$ and $n$ change with $\varepsilon_{\alpha \beta}$ as follows: when $0<\varepsilon_{\alpha \beta} \leq 0.1, n=$ 1.5 and $C=1$; when $0.1<\varepsilon_{\alpha \beta} \leq 0.2, n=3$ and $C=31.62$; when $\varepsilon_{\alpha \beta}>0.2, n=5$ and $C=790.6$. This piecewise form takes into account the nonlinear elasticity of the particles and ensures that the elastic force for large overlap is larger than in classic Hertz theory. ${ }^{1,36}$ This form is used in Section 3.1, where we investigate the influence of the drag forces on the flow curves. When inspecting the influence of the force law in Section 3.2, a simplified form of Eq. 2 is used where $C$ is set to be 1 and the value of the elastic exponent $n$ is set to be $1.5,3$ and 5 separately, which correspond to force laws with different softness.

\subsubsection{Far-field drag force $\mathrm{f}_{\alpha}^{\mathrm{far}-\mathrm{drag}}$}

The far-field drag force $\mathbf{f}_{\alpha}^{\text {far-drag }}$ on particle $\alpha$ is a hindered Stokes drag originating from the velocity difference between the imposed flow field and particle $\alpha$ :

$$
\mathbf{f}_{\alpha}^{\mathrm{far}-\mathrm{drag}}=b\left(\left\langle\mathbf{u}_{\beta}\right\rangle-\mathbf{u}_{\alpha}\right)=\frac{6 \pi R \eta_{s}}{f(\phi)}\left(\dot{\gamma} y_{\alpha} \mathbf{e}_{x}-\mathbf{u}_{\alpha}\right)
$$

It is referred to as a far-field drag term in relation with Stokesian dynamics. It has the same form as in the initial Durian model except for the expression of the dissipation 
constant in the prefactor. The latter involves the average radius of the particle $R$, the interstitial fluid viscosity $\eta_{s}$, and a term $f(\phi)$ that is the hindered settling coefficient that accounts for the reduced mobility of the particles at high volume fractions. The value of 0.01 used for this coefficient in the following is the estimated value for a suspension of spheres near close-packing. ${ }^{37}$ It can be easily shown that changing $f(\phi)$ affects the characteristic time scale for dissipation and is thus equivalent to translating the flow curves along the shear rate axis.

\subsubsection{Near-field drag force $\mathrm{f}_{\alpha \beta}^{\text {near-drag }}$}

The particles do not collide like in granular materials but come into close contact forming lubricated facets which generate elastic and near-drag forces. The near-field drag force $\mathbf{f}_{\alpha \beta}^{\text {near-drag }}$ is the tangential force acting on the flat contacting surface between neighboring particles as shown in Fig. 1b. Two different forms of near-field drag forces are used in the simulation to test the importance of the near-field contribution: an elastohydrodynamic drag force (EHD) $\mathrm{f}_{\alpha \beta}^{\mathrm{EHD}}$ and a Coulombic frictional force $\mathbf{f}_{\alpha \beta}^{\mathrm{C}}$.

The elastohydrodynamic drag force $\mathrm{f}_{\alpha \beta}^{\mathrm{EHD}}$ arises from a coupling between the hydrodynamic pressure in the lubrication film separating two particles and the elastic repulsion force between them and is given by

$$
\mathbf{f}_{\alpha \beta}^{\mathrm{EHD}}=-\left(\eta_{s} C u_{\alpha \beta, / /} E^{*} R_{c}^{3}\right)^{1 / 2} \varepsilon_{\alpha \beta}^{(2 n+1) / 4} \mathbf{n}_{\alpha \beta, / /} \cdot
$$

where $\mathbf{n}_{\alpha \beta, / /}=\mathbf{u}_{\alpha \beta, / /} /\left|\mathbf{u}_{\alpha \beta, / /}\right|$ is a unit vector parallel to the relative velocity in the direction parallel to the contact surface, $\mathbf{u}_{\alpha \beta, / /}$. This formula was originally derived for a single particle dragged along a smooth surface. ${ }^{38}$ Parameters $C, E^{*}, R \mathrm{c}$, and $\varepsilon_{\alpha \beta}$ are as defined in Eq. 2. The EHD force is computed using the difference between the 
center of mass velocities. It is to note that, in the jamming regime, the local elastic forces that trap the particles hinder their rotation, which accordingly is not considered in the simulations. ${ }^{39}$

The tangential Coulombic frictional force $\mathrm{f}_{\alpha \beta}^{\mathrm{C}}$ is defined with respect to the normal repulsive force $\mathbf{f}_{\alpha \beta}^{\text {elas }}$ assuming a simple linear relationship:

$$
\mathbf{f}_{\alpha \beta}^{\mathrm{C}}=-\mu f_{\alpha \beta}^{\mathrm{elas}} \mathbf{n}_{\alpha \beta, / /}
$$

where again $\mathbf{n}_{\alpha \beta, / /}$ is a unit vector parallel to the tangential velocity $\mathbf{u}_{\alpha \beta, / /}$. Because the contacts between soft repulsive particles like particle gels or emulsion droplets are lubricated by the solvent, it is assumed here that there is little or no static friction. ${ }^{40}$

\subsubsection{Equation of motion}

In SPGs the movement of the particles is slow and highly constrained by their jammed environment. For typical SPGs simulations, $\rho \approx 10^{3} \mathrm{~kg} / \mathrm{m}^{3}, R \approx 10^{-7} \mathrm{~m}$, $\eta_{s} \approx 10^{-3} \mathrm{~Pa} \cdot \mathrm{s}$ and the shear rate $\dot{\gamma}$ falls between $10^{-9} \mathrm{~s}^{-1}-10^{-3} \mathrm{~s}^{-1}$. The Reynolds number $\mathrm{Re}=\rho \dot{\gamma} R^{2} / \eta_{s}$ ranges from $10^{-17}$ to $10^{-11}$, and so inertia can be neglected, resulting in overdamped motion. Since all forces on each particle are balanced:

$$
\mathbf{f}_{\alpha}^{\text {far-drag }}+\sum_{\beta}\left(\mathbf{f}_{\alpha \beta}^{\text {near-drag }}+\mathbf{f}_{\alpha \beta}^{\text {elas }}\right)=\mathbf{0}
$$

The equation of motion that describes the trajectory of particle $\alpha$ is derived by expanding the $\mathrm{f}_{\alpha}^{\mathrm{far}-\mathrm{drag}}$ term in Eq. 6 using its form given by Eq. 3,

$$
\frac{d \mathbf{x}_{\alpha}}{d t}=\mathbf{u}_{\alpha}=\dot{\gamma} y_{\alpha} \mathbf{e}_{x}+M_{\alpha} \sum_{\beta}\left(\mathbf{f}_{\alpha \beta}^{\text {near-drag }}+\mathbf{f}_{\alpha \beta}^{\text {elas }}\right),
$$

where $\dot{\gamma}$ is the shear rate, $y_{\alpha}$ is the vertical location of the particle and $\mathbf{e}_{x}$ is the unit vector in the $\mathrm{x}$ direction; $M_{\alpha}=f(\phi) / 6 \pi R \eta_{s}$ is the mobility and is equivalent to the 
inverse of the dissipation constant in the Durian's model. ${ }^{24,}{ }^{25} \mathbf{f}_{\alpha \beta}^{\text {near-drag }}$ can take the form of either the elastohydrodynamic drag force $\mathrm{f}_{\alpha \beta}^{\mathrm{EHD}}$ or the Coulombic-like frictional force $\mathrm{f}_{\alpha \beta}^{\mathrm{C}}$. In the following, the flow behavior is investigated using different models of $\mathbf{f}_{\alpha \beta}^{\text {near-drag }}$ and $\mathbf{f}_{\alpha \beta}^{\text {elas }}$.

\subsection{Simulation and characterization method}

The model is implemented using a particle dynamics simulation scheme on random packings of 10,000 elastic spheres confined in a cubic box that is periodically replicated. The radii of the spheres have a $20 \%$ polydispersity to avoid forming shearinduced structures at high shear rates. ${ }^{41,42}$ Suspensions with different volume fractions are prepared as follows. A glass-like structure is first created using the compression algorithm introduced by Lubachevsky and Stillinger. ${ }^{43}$ The close-packed configurations are compressed by reducing the box size in small steps until the desired volume fraction is achieved. Because the Poisson ratio for the particles is $1 / 2$, their volume upon deformation remains constant. The volume fraction of the suspension is computed as the ratio between the total volume of the particles and the volume of the box. After each size variation, the system is allowed to relax using the conjugate gradient algorithm so there is no net force on any of the particles. This procedure ensures that each particle has reached a mechanical equilibrium and no internal stress is trapped inside the suspension. ${ }^{44}$ The volume fractions investigated range from 0.70 to 0.90 .

Constant shear rate simulations are performed using the granular package of LAMMPS. ${ }^{45}$ The shear rate is applied via Lees-Edwards boundary conditions. The position and the velocity of each particle are obtained by solving the $N$ equations of motion above using the Euler integration algorithm. ${ }^{46}$ An explicit time integration 
scheme is used to solve the equations. First, the forces on the right hand side are evaluated from the position and velocity from the last time step; then the velocity and position on the left hand side are evaluated in the current time step.

The stress tensor that characterizes the flow properties is computed from the Kirkwood formula ${ }^{47}$ :

$$
\boldsymbol{\sigma}=-\frac{1}{V} \sum_{\beta}^{N} \sum_{\alpha>\beta}^{N} \mathbf{f}_{\alpha \beta}\left(\mathbf{x}_{\alpha}-\mathbf{x}_{\beta}\right),
$$

where $V$ is the volume of simulation box, $\mathrm{f}_{\alpha \beta}$ is the total force between neighboring particles $\alpha$ and $\beta$ including near-field drag force and elastic force, $\mathbf{x}_{\alpha}$ and $\mathbf{x}_{\beta}$ are the position vectors of $\alpha$ and $\beta$. Three component of the stress are reported later in the paper: the shear stress $\sigma=\sigma_{y x}$; the first normal stress difference $N_{1}=\sigma_{x x}-\sigma_{y y}$; and the second normal stress difference $N_{2}=\sigma_{y y}-\sigma_{z z}$.

The applied dimensionless shear rate $\eta_{s} \dot{\gamma} / E^{*}$ varies from $10^{-12}$ to $10^{-4}$, depending on the specific repulsive force law. Simulations are conducted at sufficiently low shear rates to access the yield point of the suspension. All suspensions are sheared for 100 strain units to ensure that they reach steady state and that no crystallization or layering occurs. ${ }^{41,42}$ Simulations at each volume fraction and shear rate are performed starting from at least three different initial configurations of particles in the periodically replicated suspension. Each initial configuration has the requisite volume fraction and polydispersity and is statically stable, i.e., the net force on the particles is zero. It is found that the initial condition does not affect the steady state flow curves.

As values of the stresses fluctuate throughout the simulation, average stress values over the last 80 strain units are calculated for each initial configuration. Then 
the stresses obtained for the different initial configuration are averaged and the standard deviations are calculated and plotted in the figures as error bars. Some error bars are smaller than the symbols. For each volume fraction, the variations of the shear, first, and second normal stress differences with the shear rate define flow curves that are fitted to the Herschel-Bulkley equation. For each flow curve, all the data available are included in the fits and the standard deviation of the individual data of the flow curves are taken into account. The quality of the fits is characterized by the $95 \%$ confidence limits of the standard deviation reported for the parameters in the Supplemental Information (SI).

The low-frequency shear modulus $G$ is an important characteristic property of the suspension at rest. It can be computed either by subjecting the packing to an oscillatory shear deformation at small strain amplitude ${ }^{35}$ or equivalently by applying a quasi-static uniaxial stretching deformation. ${ }^{48,49}$ In this work we use the second method. The packing is deformed step by step by increasing the uniaxial deformation in small increments in a quasi-static way. At each step, the periodic box is stretched by a small amount and the particles are allowed to adjust their position with respect to their neighbors, so that they reach their local energy minimum. When the final deformation is reached, the extension ratio is $1+\delta(\delta<<1)$ and the low frequency modulus $G$ is computed from the net change in energy $\Delta U$ with respect to the undeformed state: $:^{35,48,49}$

$$
G \cong \frac{2}{3 \delta^{2}} \frac{\Delta U}{V}
$$




\section{Results}

\subsection{Static properties}

In this section, we examine the effect of the repulsive force law on the lowfrequency modulus and structural properties of SPGs. We compute the low-frequency modulus, the average contact number and the particle overlap from equilibrated packing configurations which are mechanically stable using different force laws given by Eq. 2 when $C=1$ and $n=1.5,3$, and 5. Since there is no flow, the far-field and near-field hydrodynamic contributions are not included.

\subsubsection{Low-frequency modulus}

Figure 2 shows the variation of $G$ computed for the different force laws. For each value of $n, G$ increases with the volume fraction. However, we observe significant quantitative differences between the three graphs. The variations of $G$ can be interpreted by considering that it is the product of the spring constant $k \propto \partial f_{\alpha \beta}^{\text {elas }} / \partial \varepsilon \propto \varepsilon^{n-1}$ and the number of excess contacts $Z-Z_{\mathrm{c}}$, where $Z_{\mathrm{c}}$ is the number of contacts at the jamming transition: ${ }^{50-52}$ $G \propto k\left(Z-Z_{c}\right)$ The dimensionless overlap distance $\varepsilon$ being small, the potential becomes softer when $n$ increases and the elastic modulus is smaller. $\mathcal{E}, k, Z$, and $G$ are power law
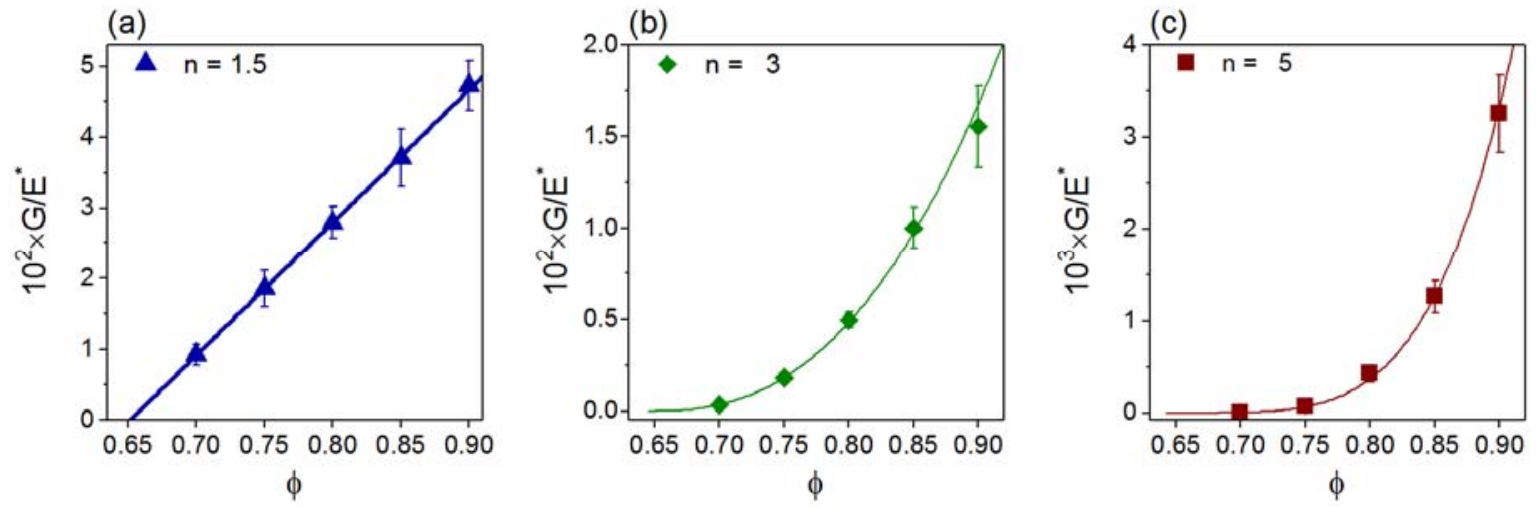

Fig. 2 Variations of the low frequency modulus $G$ versus the volume fraction for different values of the force law exponent $n$; from left to right: $n=1.5(\mathrm{a}), 3(\mathrm{~b})$, and 5 (c). The continuous lines are fits of the data to expression (10d) with $\gamma=1\left(\phi_{\mathrm{c}}=0.651 \pm 0.001\right), \gamma=2.5\left(\phi_{\mathrm{c}}=0.645 \pm 0.002\right), \gamma=4.5\left(\phi_{\mathrm{c}}=0.641 \pm 0.002\right)$ for (a) to (c), respectively. 
functions of the distance to the jamming point: ${ }^{48,50}$

$$
\begin{aligned}
& \varepsilon=\varepsilon_{0}\left(\phi-\phi_{c}\right), \\
& k=k_{0}\left(\phi-\phi_{c}\right)^{n-1}, \\
& Z-Z_{c}=Z_{0}\left(\phi-\phi_{c}\right)^{\zeta}, \\
& G=G_{0}\left(\phi-\phi_{c}\right)^{\gamma},
\end{aligned}
$$

where $\gamma=n+\zeta-1 ; \phi_{\mathrm{c}}$ is the jamming volume fraction; $Z_{\mathrm{c}}$ is the average contact number at the jamming point; $\varepsilon_{0}, k_{0}$, and $Z_{0}$ are prefactors. For monodisperse packings and in the asymptotic limit of large systems, the exponent $\zeta$ is equal to $1 / 2, Z_{\mathrm{c}}=6$, and $\phi_{\mathrm{c}} \cong$ 0.64 .

The elastic moduli shown in Fig. 2 are well-fitted to Eq. 10d with $\gamma=1,2.5$, and 4.5, which are the values of $\gamma$ expected for $n=1.5,3$, and 5 respectively. Letting the jamming point $\phi_{\mathrm{c}}$ be a fitted parameter, we find that it consistently occurs at $\phi_{\mathrm{c}}=0.65 \pm 0.01$. Note that the value of $\phi_{\mathrm{c}}$ is larger for polydisperse than for monodisperse suspensions. The details of the fitting parameters are presented in Supplemental Information.

\subsubsection{Structural properties of SPGs for different elastic force laws}

Figures 3a-b show the variations of the contact number $Z$ and the dimensionless average overlap distance $\varepsilon$ versus the volume fraction for different values of the exponent $n$ in equilibrated packings. Because the elastic repulsive forces are smaller for greater values of the exponent $n$, packings with greater $n$ have larger average overlap distances for the same volume fraction. To compensate for larger overlap and still have the same volume fraction, they thus have fewer average contacts. 

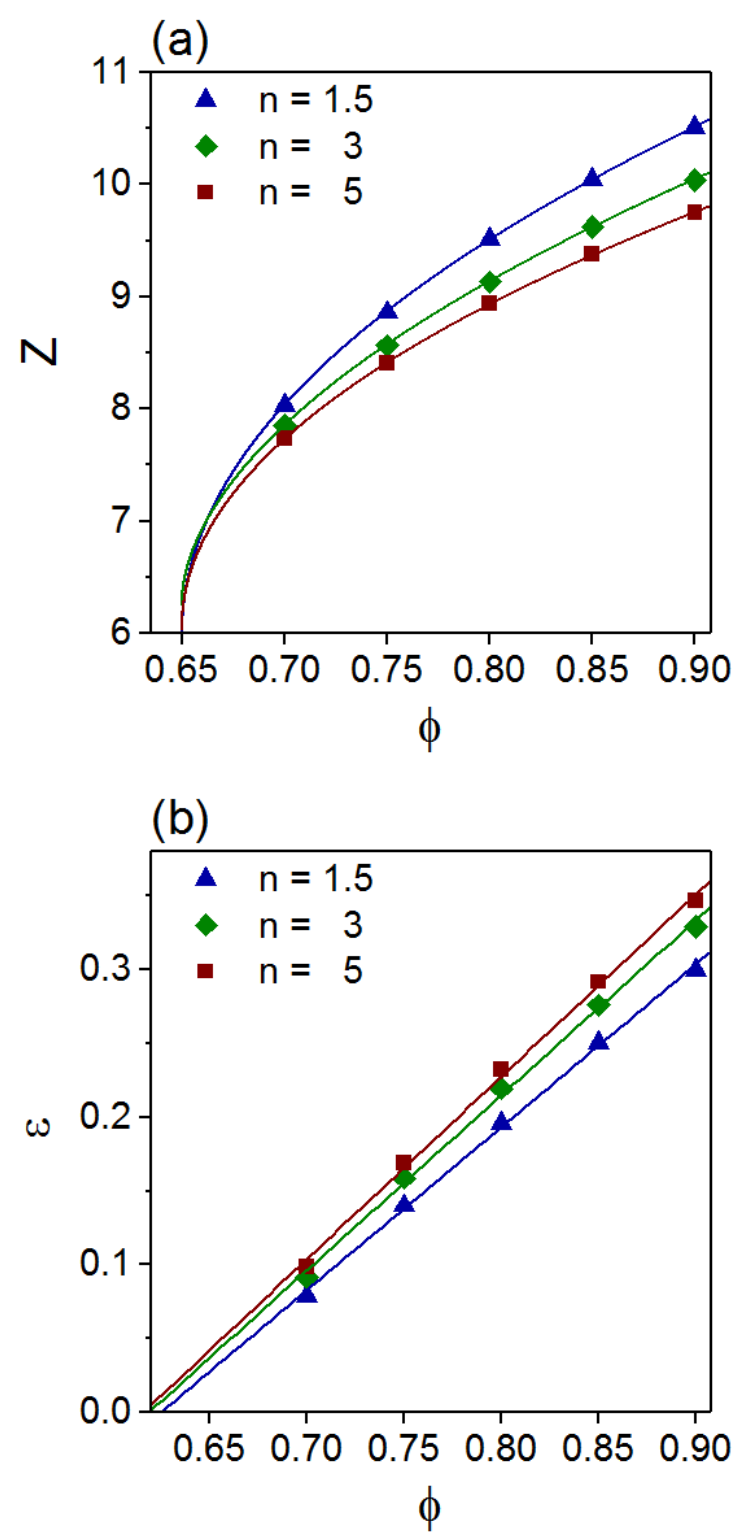

Fig. 3 Variations of the average contact number $Z$ (a) and overlap distance $\varepsilon$ (b) for different values of exponent $n$. The solid lines in (a) are fits to Eq. $10 \mathrm{c}$ with $\phi_{\mathrm{c}}=0.65$ yielding: $Z_{\mathrm{c}}=6.0 \pm 0.1,6.3 \pm 0.1,6.0 \pm 0.1$ and $\zeta=0.49 \pm 0.02,0.54 \pm 0.02,0.49 \pm 0.02$, for $n=1.5,3,5$ respectively. The solid lines in (b) are fits to Eq. $10 \mathrm{a}$ yielding: $\phi_{\mathrm{c}}=0.63 \pm 0.01(n=1.5) ; \phi_{\mathrm{c}}=0.62 \pm 0.01(n=3) ; \phi_{\mathrm{c}}=0.62 \pm 0.01(n=5)$.

In Fig. 3a we have fitted the data to Eq. 10c assuming that jamming occurs at $\phi_{\mathrm{c}}=0.65$, the value which was determined from the shear modulus variations in Fig. 2 . The data are well represented by Eq. 10c. We find that the average number of contacts at $\phi_{\mathrm{c}}$ is approximately six and the exponent $\zeta$ is close to 0.5 , which are the values expected for monodisperse suspensions. ${ }^{4,50}$ The fitting parameters are given in the SI. Finally, we have fitted the data for the average overlap distance in Fig. $3 b$ to the linear 
expression given by Eq. 10a. The resulting values of $\phi_{\mathrm{c}}$ are slightly different from those previously determined but both determinations remain within the interval of confidence which is admissible (see also SI Section).

\subsection{The effect of the near-field drag force on the flow properties}

In this section we investigate the effect of near-field contributions on the shear stress, first and normal stress differences. The elastic force law is has the generalized Hertz form in Eq. 2 and the far-field Stokes force is included in the simulations.

\subsubsection{Elastohydrodynamic (EHD) drag force}

Figures 4a-c show the results of the dimensionless shear stress $\sigma$, the first normal stress difference $N_{1}$ and the second normal stress difference $N_{2}$ versus the dimensionless shear rate at different volume fractions with and without EHD drag forces. All three quantities are well-fitted to Herschel-Bulkley equations over the entire range of shear rates investigated. It is also interesting to note that the first and second normal stress differences $N_{1}$ and $N_{2}$ are similar in magnitude and opposite in sign for the same volume fraction at the same shear rate. So-called film fluids, such as emulsions and foams, exhibit this property due to the storage of elastic energy in their interfaces ${ }^{53}$. Here, the elastic energy is stored in the local deformations at contact.

Interestingly, the results show little difference between the model with EHD drag forces and the one without EHD drag forces, which demonstrate the non-essential role of the near-field EHD drag forces in predicting shear stress and normal stress differences in the range of shear rates considered. 

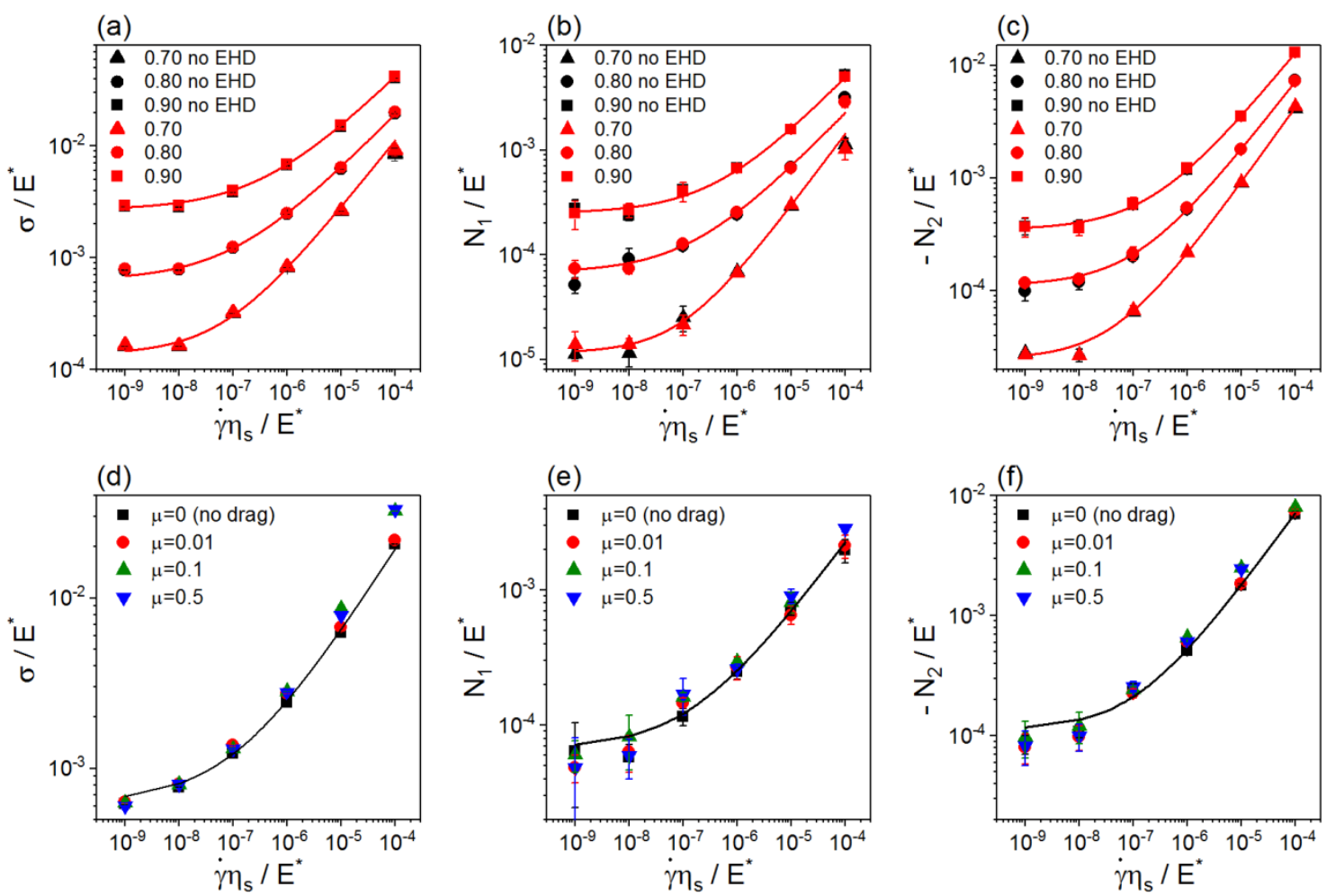

Fig. 4 Top: role of the near-field elastohydrodynamic force; dimensionless shear stress (a), first normal stress difference (b) and second normal stress difference (c) versus dimensionless shear rate at $\phi=0.70,0.80$ and 0.90 with EHD (red symbols) and without (black symbols). Bottom: role of Coulombic drag forces; dimensionless shear stress (d), first normal stress difference (e), and second normal stress difference (f) versus dimensionless shear rate at $\phi=0.8$ with coefficient $\mu=0,0.01,0.1,0.5$. The results for $\phi=0.70$ and 0.90 also show negligible difference for different values of $\mu$ at $\phi=0.80$ and are not shown for clarity. Solid lines are fitted curves for simulations with no drag forces to the Herschel-Bulkley equations $\sigma / E^{*}=\sigma_{y} / E^{*}+k_{\sigma}\left(\dot{\gamma} \eta_{s} / E^{*}\right)^{m}$ and $N_{i} / E^{*}=N_{i y} / E^{*}+k_{N_{i}}\left(\dot{\gamma} \eta_{s} / E^{*}\right)^{m_{i}}$ with parameters reported in SI $\left(N_{i}\right.$ refers to $N_{1}$ or $\left.-N_{2}\right)$.

\subsubsection{Coulombic frictional drag force}

Figures 4d-f show the results for the dimensionless shear stress and dimensionless normal stress differences versus the dimensionless shear rate at $\phi=0.8$ when Coulombic frictional drag forces with different friction coefficients $\mu$ are included in the simulations. Four cases are tested with $\mu$ set to be $0,0.01,0.1$ or 0.5 . The results show little difference among flow curves for all the friction coefficients. Similar to the near-field EHD, Coulombic frictional drag forces play a negligible role in determining the shear stress versus shear rate flow curve. 


\subsection{The effect of the repulsive force law on the flow properties}

In this section we investigate the effect of the repulsive force law on the shear stress, first and normal stress differences. The force law is given by Eq. 2 with $C=1$ and $n=1.5,3$, and 5. Only the far-field Stokes force is included in the simulations; the near-field contributions being negligible from the previous section are not included.

\subsubsection{Shear stress and first and second normal stress differences}

The flow curves computed for exponents $n=1.5,3$ and 5 at different volume fractions are shown in Figs. 5a-c. At large shear rates and low values of the shear modulus, a microstructural transition could be observed, where the initially disordered suspension was evolving to a partially ordered structure with layers parallel to the flow-vorticity plane. ${ }^{41}$ Those corresponding shear stress data are not plotted in Fig. 5. For the same volume fraction, shear stresses are larger for smaller $n$. Large shear stresses are generated for large overlap distances which occur for large volume fractions and small $n$. The corresponding dimensionless first and second normal stress differences in Figs 5d-f and Figs 5g-i show the same trend as the shear stresses. They all exhibit a yield normal stress at small shear rates. Second normal stress differences $N_{2}$ are slightly larger in magnitude than $N_{1}$ and opposite in sign. The differences between $N_{1}$ and $N_{2}$ are greater for lower volume fraction, higher shear rates and larger $n$. Microstructures and pair distribution functions for different $n$ are also investigated, which exhibit the same qualitative behavior as reported earlier. ${ }^{11,35,52}$ Irrespective of the interparticle force law, the flow curves can be fitted to the canonical HerschelBulkley equation. The parameters $m, \sigma_{y}$ and $k$ as well as pair distribution functions are reported in the SI. The exponent $m$ lies in the range between 0.4 and 0.5 with weak dependencies on the volume fraction and the exponent of the force law. The exponent $m$ decreases slightly when the volume fraction increases. This trend has also been 

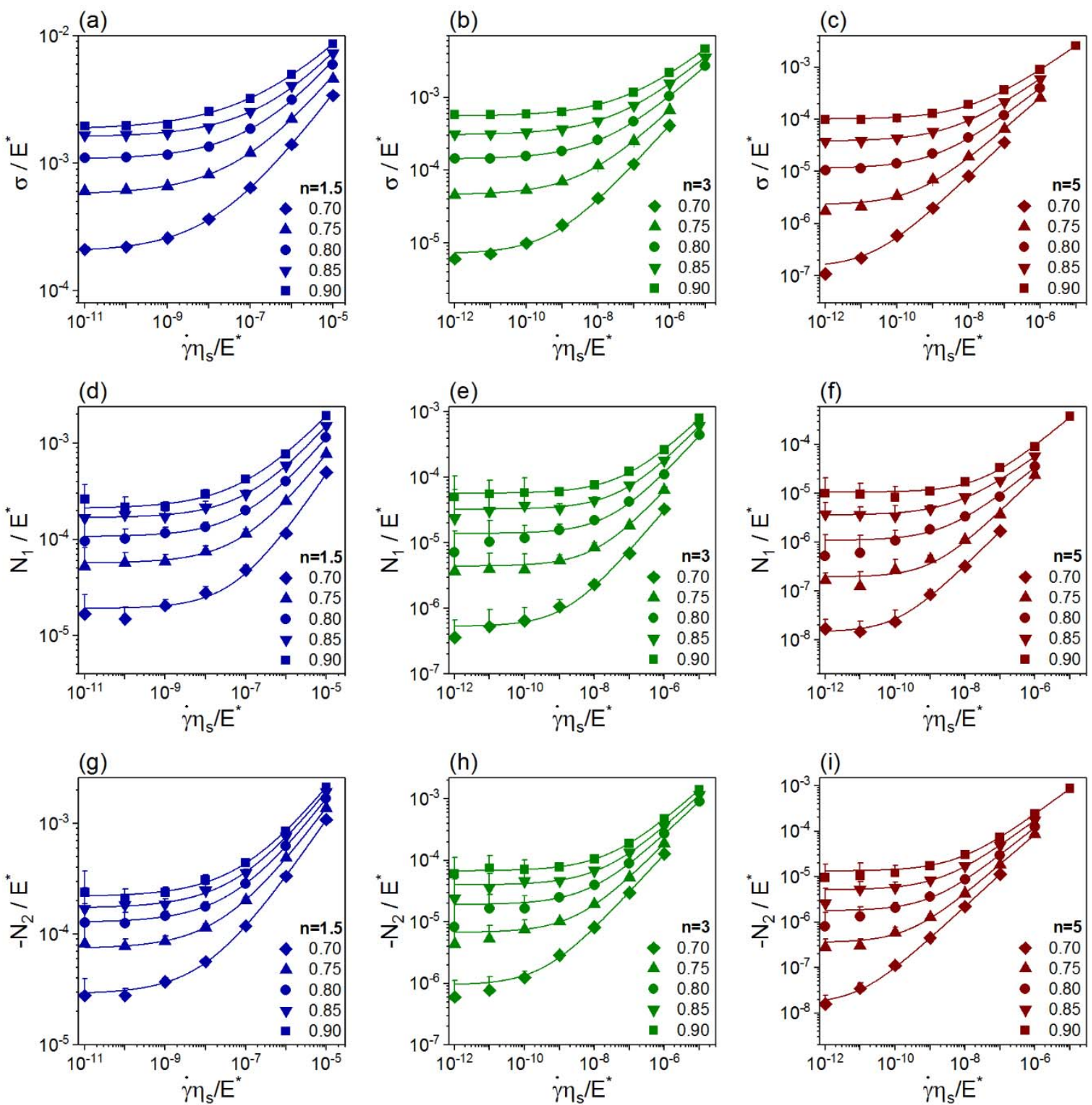

Fig. 5 Dimensionless shear stress (a-c), dimensionless first (d-f) and second normal stress differences (g-i) versus dimensionless shear rate for different values of exponent $n=1.5,3,5$. Solid lines are fitted curves to the Herschel-Bulkley equation. Only positive parts of error bars are shown for easier visualization. Data for all Herschel-Bulkley parameters are listed in SI.

noted in experiments. ${ }^{4}$ The softness of the interaction has a small influence on the Herschel-Bulkley exponent, which has its smallest value for the Hertz law with $n=1.5$ and is closer to 0.5 for $n=3$ and 5. The same trends are observed for the first and second normal stress differences, although the Herschel-Bulkley exponents are slightly larger than for the stress and close to 0.5 . 


\subsubsection{Scaling of shear stress and first and second normal stress differences}

The shear stresses and normal stresses for each volume fraction and each elastic force law ( $n=1.5,3$ and 5 ) can be collapsed onto universal flow curves by scaling the stress with the yield stress and the shear rate with $G / \eta_{s}$, as shown in Fig. 6. The collapse is satisfactory except for the shear stress data at volume fraction $\phi=0.7$ close to the jamming transition, which fall slightly outside the master curve. These data are not included in the following fits. Each master curve can be described by an equation of the Herschel-Bulkley form:

$$
\begin{gathered}
\sigma / \sigma_{y}=1+\tilde{k}_{\sigma}\left(\dot{\gamma} \eta_{s} / G\right)^{\widetilde{m}} \\
N_{1} / \sigma_{y}=\widetilde{N}_{1 y}+\tilde{k}_{N_{1}}\left(\dot{\gamma} \eta_{s} / G\right)^{\widetilde{m}_{1}} \\
-N_{2} / \sigma_{y}=-\widetilde{N}_{2 y}+\widetilde{k}_{N_{2}}\left(\dot{\gamma} \eta_{s} / G\right)^{\widetilde{m}_{2}}
\end{gathered}
$$

The fitting parameters are tabulated in the SI.

For the shear stress, the exponent of the Herschel-Bulkley power law $\widetilde{m}$ ranges from 0.41 to 0.50 as $n$ increases from 1.5 to 5 . For comparison the dotted lines in Figs. 6a-c represent the best fit to experimental data obtained for microgel suspensions, ${ }^{1,7}$ yielding a Herschel-Bulkley exponent of 0.45 . The fitted experimental data match the simulated universal flow curve reasonably well for $n=1.5$ and 3 . The agreement with experiments is not as good for $n=5$, the simulated data being above the experimental lines at the larger shear rates. For the first and second normal stress differences, the Herschel-Bulkley exponents $\widetilde{m}_{1}$ and $\widetilde{m}_{2}$ range from 0.53 to 0.62 as $n$ increases from 1.5 to 5. For each force law, the Herschel-Bulkley exponents are about the same for $N_{1}$ and $N_{2}$. We observed that the error bars at low shear rates are large because of the large fluctuations relative to $N_{1 y}$ and $N_{2 y}$. Finally it is interesting to note that the yield normal stresses $N_{1 y}$ and $N_{2 y}$ are about an order of magnitude smaller than the yield shear stress. 
(a)
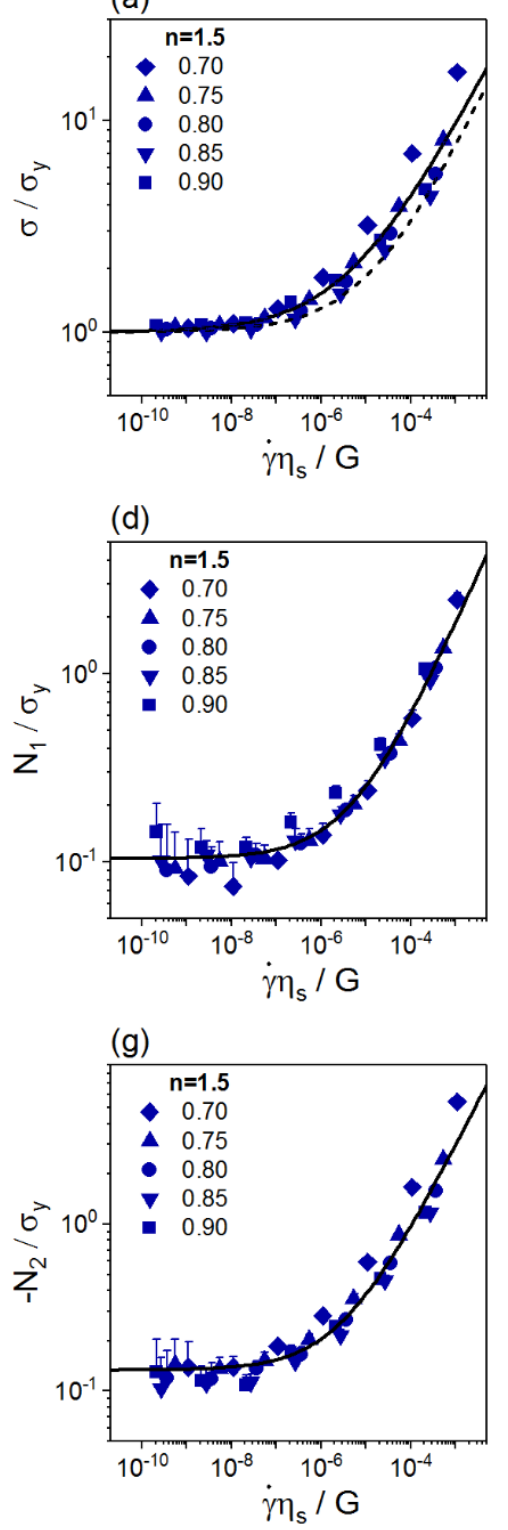

(b)
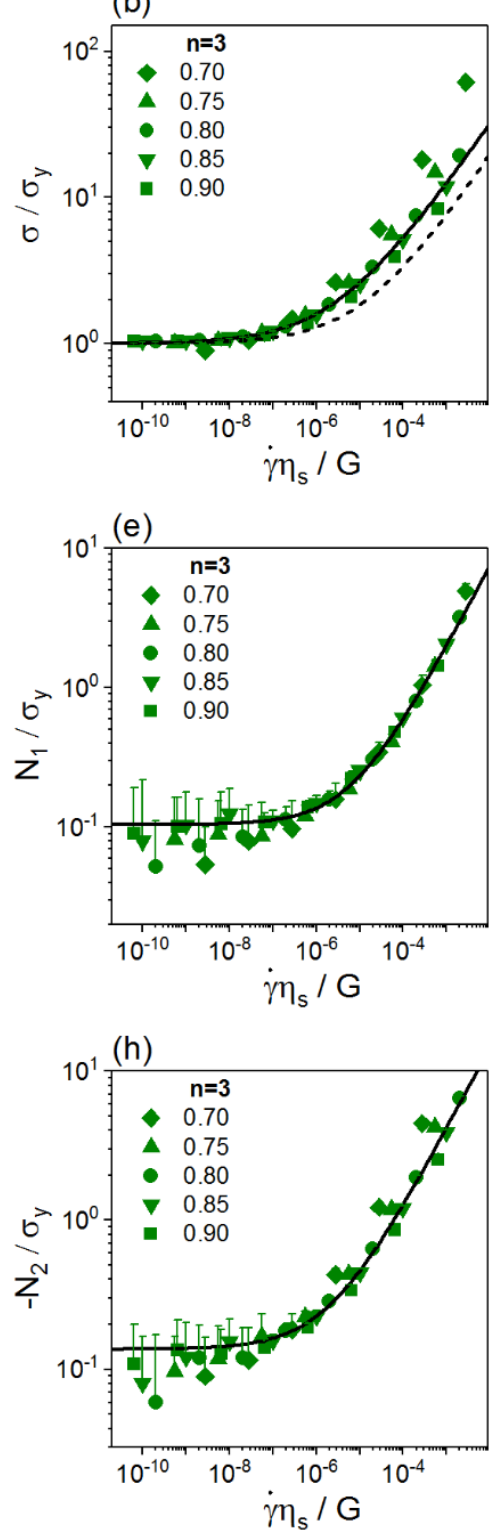
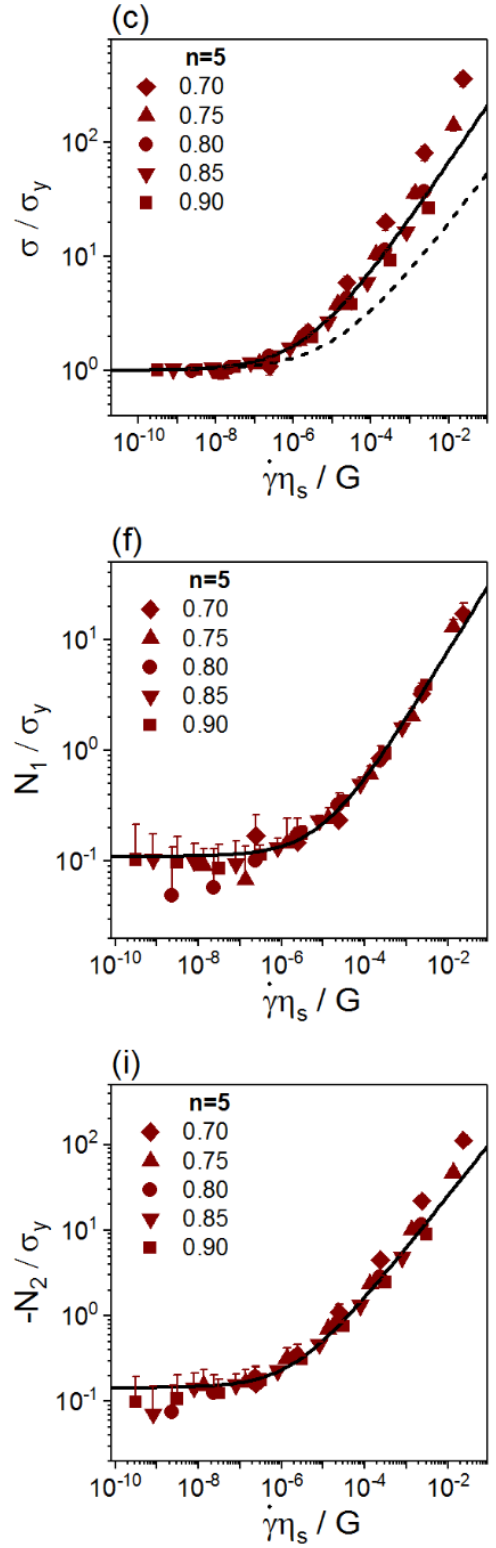

Fig. 6 (a-c): Collapse of flow curves for elastic exponent $n=1.5,3$, and 5(c). The solid lines are the fitted Herschel-Bulkley curves; the dashed lines of equation $\sigma / \sigma_{y}=1+150\left(\dot{\gamma} \eta_{s} / G\right)^{0.45}$ are fits to collapsed experimental data of microgels. ${ }^{4,7}$ (d-i): Collapse of first and second normal stress differences for $n=1.5,3,5$. Only the upper halves of the error bars are shown for easier visualization. All Herschel-Bulkley parameters are listed in the SI.

\section{Discussion}

Our results demonstrate that the elastohydrodynamic and Coulombic near-field drag are not important for predicting the rheology of SPGs. In our previous studies, we already observed that only the elastic contact forces contribute significantly to the shear and normal stresses for SPGs. ${ }^{11,35}$ In those simulations, the near-field drag was 
included, which might influence the microstructure and so indirectly the rheology. The results presented in Fig. 2 show that removing the near-field drag altogether has no substantial effect on the microstructure and the rheology. However, the result that the flow curves computed at different volume fractions or measured under different experimental conditions collapse when the shear rate is rescaled with $\dot{\gamma} \eta_{s} / G$ indicate that the viscous drag forces acting on the soft particles are important and that the shear rheology is clearly determined in part by the viscosity of the interstitial fluid. ${ }^{1,7,11}$ In the simulations this effect is captured by the far-field drag force acting on the particles (Eq. 3).

It is noteworthy that the dimensionless shear rate $\dot{\gamma} \eta_{s} / G$ is effective in collapsing the shear stress and normal stress differences for different volume fractions for all the pairwise potentials considered in this study. Since many repulsive interactions in practice fall within this range of potentials, one could expect it to be quite generic. Indeed, previously, this dimensionless shear rate has been used to successfully create master flow curves from experimental data on systems as different as microgels, concentrated emulsions, and star polymers. ${ }^{1,7,54}$ The dimensionless shear rate $\dot{\gamma} \eta_{s} / G$ is the ratio of the time scale $1 / \dot{\gamma}$ associated with the shear deformation, to the characteristic time $\eta_{\mathrm{s}} / G$, which expresses the competition between the cage elasticity and the viscous forces acting on the particles when they rearrange. The volume fraction dependence of the characteristic time $\eta_{\mathrm{s}} / G$ is embodied in the shear modulus. The dimensionless shear rate $\dot{\gamma} \eta_{s} / G$ controls several other important dynamical phenomena in SPGs. First the shear induced crystallization of monodispersed SPGs or layering of polydisperse SPGs at different volume fractions occurs at some critical value of $\dot{\gamma} \eta_{s} / G^{41,42}$ In another context, the initial short time relaxation of the stress upon flow cessation is driven by a characteristic time which 
involves the dissipation time $\eta_{s} / E^{*}$ and the dimensionless shear rate $\dot{\gamma} \eta_{s} / G$ applied during the initial flow. ${ }^{55}$ The resulting residual stresses trapped into SPGs are also controlled by $\dot{\gamma} \eta_{s} / G{ }^{44,55}$ This scaling highlights the importance of the competition between viscous and elastic forces in sheared SPGs. The scaling factors $\sigma_{\mathrm{y}}$ and $\eta_{\mathrm{s}} / G$ in Fig. 6 can be expressed as functions of $\phi-\phi_{c}$. From our simulation data, the yield stress varies like $\sigma_{y} \sim\left(\phi-\phi_{c}\right)^{n}$ (see Fig. S2 in the SI), which has also been noted by others. ${ }^{56,57}$ The characteristic time $\eta_{\mathrm{s}} / G$ used to scale the shear rate vary like $(\phi-$ $\left.\phi_{c}\right)^{1 / 2-n}$. Thus for Hertz potential we have: $\eta_{s} / G \sim\left(\phi-\phi_{c}\right)^{-1}$, which differs from the scaling factors usually found near the jamming transition. ${ }^{10,12,28,29,56,57}$ This shows that the scaling properties we propose are valid far from the jamming transition.

When the elastic repulsion between the particles obeys to the Hertz potential ( $C=1$ and $n=1.5$ in Eq. 2 ), there exists an alternative non-dimensionalization of the shear rate which collapses the flow curves onto master curves. Indeed in Fig. 5a, the flow curves are of the form $\sigma / E^{*}=\sigma_{y} / E^{*}+k_{\sigma}\left(\dot{\eta}_{s} / E^{*}\right)^{m}$ and the prefactors $k_{\sigma}$ are found proportional to $G / E^{*}$ (see SI) Moreover the yield stress and the elastic modulus are related through $\sigma_{y}=G \gamma_{y}$, where $\gamma_{y}$ is the yield strain. The same reasoning holds for the first and second normal stress differences. It follows that the shear stress and normal stress differences at different volume fractions must be correlated using the equations:

$$
\begin{gathered}
\sigma / \sigma_{y}=1+\bar{k}_{\sigma}\left(\dot{\eta} \eta_{s} / \gamma_{y}^{2} E^{*}\right)^{\bar{m}} \\
N_{1} / \sigma_{y}=\bar{N}_{1 y}+\bar{k}_{N 1}\left(\dot{j} \eta_{s} / \gamma_{y}^{2} E^{*}\right)^{\bar{m}_{1}} \\
-N_{2} / \sigma_{y}=-\bar{N}_{2 y}+\bar{k}_{N 2}\left(\dot{\gamma} \eta_{s} / \gamma_{y}^{2} E^{*}\right)^{\bar{m}_{2}}
\end{gathered}
$$


Fig. 7 shows the normalized shear and normal stresses at different volume fractions on a master curve as a function of the dimensionless shear rate $\dot{\gamma} \eta_{s} / \gamma_{y}^{2} E^{*}$. The new fitting parameters are listed in the SI. Now, the yield strain embodies the volume fraction dependence of the rescaled shear rate enabling a master flow curve. While this alternative scaling works well for $n=1.5$, it fails for $n=3$ or 5 because for these potentials, the consistency parameters in the Herschel-Bulkley equations for the stress and normal stress differences do not vary linearly with $G / E^{*}$. It is interesting to note that the non-dimensional parameters used in Eqs. (11) and (12) simply differ by the ratio $G / \gamma_{y}^{2}$, which is found constant in our simulations. This follows from the scaling of $G$ and $\sigma_{y}$ with $\phi-\phi_{c}$. As noted previously we have: $G \sim\left(\phi-\phi_{c}\right)^{n-1 / 2}$ and $\sigma_{y} \sim\left(\phi-\phi_{c}\right)^{n}$. Since $\gamma_{y}=\sigma_{y} / G$, it follows: $G / \gamma_{y}^{2} \sim\left(\phi-\phi_{c}\right)^{n-3 / 2}$. For $n=1.5, G / \gamma_{y}^{2}$ is a constant independent of volume fraction, but not so for other values of $n$. This alternative non-dimensionalization of the shear rate was noted earlier by Seth et al. ${ }^{11}$ in simulations using the generalized Hertz potential given by Eq. 2 with varying $C$ and $n$ depending on the overlap of the particles, ${ }^{11}$ and in experiments.. ${ }^{4,11}$ The fact that it worked for this modified potential indicates that the pairwise interactions with the modified potentials and in experiments are close to that of the classic Hertz potential.
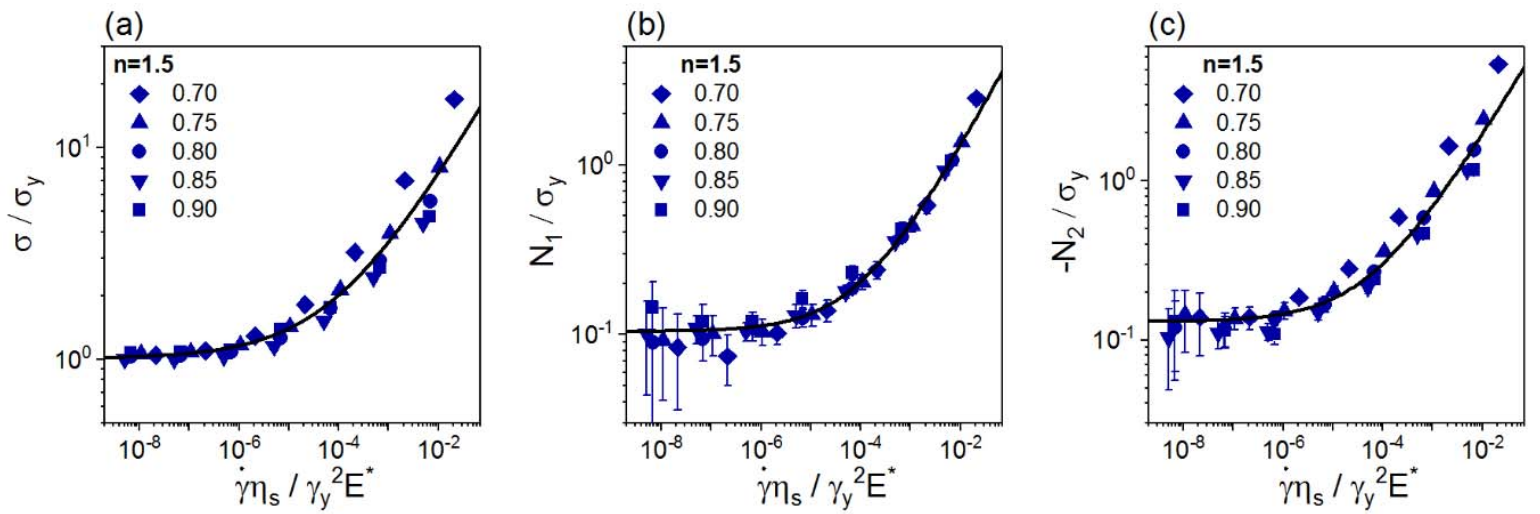

Fig. 7 Collapse of all shear stresses and normal stress differences with $\dot{\gamma} \eta_{s} / \gamma_{y}^{2} E^{*}$ for $n=1.5$. The solid lines are Herschel-Bulkley fits to the data. The fitting parameters are listed in the SI. 
Another question of interest concerns the value of the Herschel-Bulkley exponents, which for the shear stress and normal stress differences are close to 0.5 . However a close examination of the results indicates a systematic variation that signals some effect of the potential. Indeed in the master curves of Fig. 6, which include data for the entire range of volume fractions investigated, the Herschel-Bukley exponents for the collapsed shear stress data are $0.41,0.43$, and 0.51 for $n=1.5,3$ and 5, respectively. A similar increase of the exponents with $n$ is observed for the first and second normal stress differences. The variations are outside the confidence intervals showing that stiffer potentials yield slightly larger Herschel-Bulkley exponents. This trend is supported by the fact that simulations using the generalized Hertz law, which progressively becomes stiffer as the particle compression is increased, yield exponents equal to 0.50 instead of 0.41 for the simple Hertz law. ${ }^{11}$ In experiments, the slight dependence of the exponent on the potential may explain the difference between concentrated emulsions $^{1,11}(m=0.50)$ and microgel suspensions ${ }^{7}(m=0.45)$. It has been proposed that the physical origin of this non trivial value is associated to nonaffine deformations that occur through individual rearrangements localized in time and space. In elastoplastic models the dynamics of long-ranged stress and strain fluctuations in the surrounding of a localized yielding event is accounted for by a Fokker-Planck equation which predicts exponents in the range of $0.50-0.59 .{ }^{19,22,23}$ In this context, our results suggest that avalanches and cascade rearrangements can be affected by the exact shape of the elastic potential.

\section{Conclusions}

A computational study has been performed to show the universal form of the flow curve for soft particle glasses with different pairwise elastic and frictional forces. 
It is found that pairwise or near-field viscous and Coulombic sliding forces play no significant role in terms of the macroscopic rheology of these materials. The rheology is dominated by the elastic forces acting normally between the particles. For a given pairwise elastic interaction potential, the variations of the shear and normal stresses can be collapsed for all volume fractions by rescaling the stress by the yield stress and the shear rate by the characteristic time $\eta_{s} / G$, the ratio of the suspending viscosity and low frequency shear modulus. This is equivalent to another scaling proposed earlier, which involves the yield stress and the characteristic time $\eta_{s} / \gamma_{y}^{2} E^{*} .{ }^{11}$ The advantage of using the former scaling over the latter is that it does not required the knowledge of the particle contact modulus, which is generally difficult to measure directly. Inversely the second scaling provides an indirect characterization of the particle modulus. ${ }^{4}$ These scaling forms are valid deep into the jammed phase. The exponent for the HerschelBulkley fits for these flow curves ranges from about 0.4 to 0.6 and increases with the exponent of the elastic interaction. For a given elastic potential, there is a universal representation of the flow curves for SPGs for all volume fractions. These results highlight the essential and subtle role played by elastic forces in the flow properties of SPGs. The next step is to connect this result concerning the macroscopic behavior of SPGs to the microscopic dynamics, which will be the subject of a forthcoming publication.

\section{Acknowledgements}

FK and RTB gratefully acknowledge partial support from NSF Grant CBET 1336852. TL and RTB gratefully acknowledge support from the William and Bettye Nowlin Chair of Engineering at UT Austin. 


\section{References}

1. R. T. Bonnecaze and M. Cloitre, Adv. Polym. Sci., 2010, 236, 117-161.

2. J. B. Thorne, G. J. Vine and M. J. Snowden, Colloid. Polym. Sci., 2011, 289, 625-646.

3. D. Vlassopoulos and M. Cloitre, Curr. Opin. Colloid Interface Sci., 2014, 19, 561-574.

4. $\quad$ C. Pellet and M. Cloitre, Soft Matter, 2016, 12, 3710-3720.

5. F. Scheffold, F. Cardinaux and T. G. Mason, J. Phys.: Condens. Matter, 2013, 25, 502101.

6. D. Bonn, M. M. Denn, L. Berthier, T. Divoux and S. Manneville, Rev. Mod. Phys., 2017, 89, 035005.

7. $\quad$ M. Cloitre, R. Borrega, F. Monti and L. Leibler, Phys. Rev. Lett., 2003, 90, 068303.

8. $\quad$ L. Becu, S. Manneville and A. Colin, Phys. Rev. Lett., 2006, 96, 138302.

9. N. D. Denkov, S. Tcholakova, K. Golemanov, K. P. Ananthapadmanabhan and A. Lips, Phys. Rev. Lett., 2008, 100, 138301.

10. K. N. Nordstrom, E. Verneuil, P. E. Arratia, A. Basu, Z. Zhang, A. G. Yodh, J. P. Gollub and D. J. Durian, Phys. Rev. Lett., 2010, 105, 175701.

11. J. R. Seth, L. Mohan, C. Locatelli-Champagne, M. Cloitre and R. T. Bonnecaze, Nat. Mater., 2011, 10, 838-843.

12. J. Paredes, M. A. J. Michels and D. Bonn, Phys. Rev. Lett., 2013, 111, 015701.

13. A. Basu, Y. Xu, T. Still, P. Arratia, Z. Zhang, K. Nordstrom, J. M. Rieser, J. Gollub, D. Durian and A. Yodh, Soft Matter, 2014, 10, 3027-3035.

14. J. Lin, E. Lerner, A. Rosso and M. Wyart, Proc. Natl. Acad. Sci. U. S. A., 2014, 111, 14382-14387.

15. J. S. Langer, Phys. Rev. E, 2015, 92, 012318.

16. P. Sollich, Phys. Rev. E, 1998, 58, 738-759.

17. P. Hébraud and F. Lequeux, Phys. Rev. Lett., 1998, 81, 2934-2937.

18. A. Nicolas and J. L. Barrat, Phys. Rev. Lett., 2013, 110, 44003.

19. L. Bocquet, A. Colin and A. Ajdari, Phys. Rev. Lett., 2009, 103, 036001.

20. A. Nicolas, K. Martens and J.-L. Barrat, EPL (Europhysics Letters), 2014, 107, 44003.

21. F. Puosi, J. Olivier and K. Martens, Soft Matter, 2015, 11, 7639-7647.

22. J. Lin and M. Wyart, Phys. Rev. E, 2018, 97, 012603.

23. E. Agoritsas and K. Martens, Soft Matter, 2017, 13, 4653-4660.

24. D. J. Durian, Phys. Rev. Lett., 1995, 75, 4780-4783.

$25 . \quad$ D. J. Durian, Phys. Rev. E, 1997, 55, 1739-1751.

26. A. Lemaître and C. Caroli, Phys. Rev. Lett., 2009, 103, 065501.

27. V. J. Langlois, S. Hutzler and D. Weaire, Phys. Rev. E, 2008, 78, 021401.

28. B. P. Tighe, E. Woldhuis, J. J. C. Remmers, W. van Saarloos and M. van Hecke, Phys. Rev. Lett., 2010, 105, 088303 .

29. P. Olsson and S. Teitel, Phys. Rev. Lett., 2012, 109, 108001

30. P. Chaudhuri, L. Berthier and L. Bocquet, Phys. Rev. E, 2012, 85, 021503.

31. V. Mansard, A. Colin, P. Chaudhuri and L. Bocquet, Soft Matter, 2013, 9, 7489-7500.

32. C. Fusco, T. Albaret and A. Tanguy, Eur. Phys. J. E, 2014, 37, 43.

33. M. Gross, T. Krüger and F. Varnik, Soft Matter, 2014, 10, 4360-4372.

34. V. V. Vasisht, S. K. Dutta, E. Del Gado and D. L. Blair, Phys. Rev. Lett., 2018, 120, 018001.

35. L. Mohan, C. Pellet, M. Cloitre and R. Bonnecaze, J. Rheol., 2013, 57, 1023-1046.

36. K. K. Liu, D. R. Williams and B. J. Briscoe, J. Phys. D: Appl. Phys., 1998, 31, 294-303.

37. R. Phillips, J. Brady and G. Bossis, Phys. Fluids, 1988, 31, 3462-3472.

38. J. R. Seth, M. Cloitre and R. T. Bonnecaze, J. Rheol., 2008, 52, 1241-1268.

39. C. Dagallier, F. Cardinaux, H. Dietsch and F. Scheffold, Soft Matter, 2012, 8, 4067-4071.

40. J. Gong, Y. Iwasaki, Y. Osada, K. Kurihara and Y. Hamai, J. Phys. Chem. B, 1999, 103, 6001-6006.

41. F. Khabaz, T. Liu, M. Cloitre and R. T. Bonnecaze, Phys. Rev. Fluids, 2017, 2, 093301.

42. $\quad$ F. Khabaz, M. Cloitre and R. T. Bonnecaze, Phys. Rev. Fluids, 2018, 3, 033301.

43. B. D. Lubachevsky and F. H. Stillinger, J. Stat. Phys., 1990, 60, 561-583.

44. L. Mohan, R. T. Bonnecaze and M. Cloitre, Phys. Rev. Lett., 2013, 111, 268301.

45. S. Plimpton, J. Comput. Phys., 1995, 117, 1-19.

46. D. C. Rapaport, The art of molecular dynamics simulation, Cambridge University Press, Cambridge, UK ; New York, NY, 2nd edn., 2004.

47. R. G. Larson, The structure and rheology of complex fluids, Oxford University Press, New York, 1999.

48. M. D. Lacasse, G. S. Grest, D. Levine, T. G. Mason and D. A. Weitz, Phys. Rev. Lett., 1996, 76, 3448-3451.

49. J. R. Seth, M. Cloitre and R. T. Bonnecaze, J. Rheol., 2006, 50, 353-376.

50. C. S. O'Hern, L. E. Silbert, A. J. Liu and S. R. Nagel, Phys. Rev. E, 2003, 68, 011306.

51. M. van Hecke, J. Phys.: Condens. Matter, 2010, 22, 033101.

52. L. Mohan and R. T. Bonnecaze, Soft Matter, 2012, 8, 4216-4222.

53. R. Larson, J. Rheol., 1997, 41, 365-372.

54. B. M. Erwin, M. Cloitre, M. Gauthier and D. Vlassopoulos, Soft Matter, 2010, 6, 2825-2833.

55. L. Mohan, M. Cloitre and R. T. Bonnecaze, J. Rheol., 2015, 59, 63-84.

56. M. Dinkgreve, J. Paredes, M. Michels and D. Bonn, Phys. Rev. E, 2015, 92, 012305.

57. P. Olsson and S. Teitel, Phys. Rev. E, 2011, 83, 030302. 


\title{
Supplementary Information
}

\section{On the universality of the Flow Curve for Soft-Particle Glasses}

\author{
Tianfei Liu, ${ }^{\mathrm{a}}$ Fardin Khabaz, ${ }^{\mathrm{a}}$ Michel Cloitre ${ }^{\mathrm{b}}$ and Roger T. Bonnecaze ${ }^{\mathrm{a}}$
}

${ }^{a}$ McKetta Department of Chemical Engineering and Texas Materials Institute, University of Texas at Austin, Austin, TX 78712 USA

${ }^{\mathrm{b}}$ Soft Matter and Chemistry, CNRS, ESPCI Paris, PSL Research University, 10 Rue Vauquelin, 75005

Paris, France

\section{Pair distribution function for suspension at rest for different force laws.}

The pair distribution function for suspensions with different pairwise elastic force laws are similar at rest, as noted in Fig. S1.

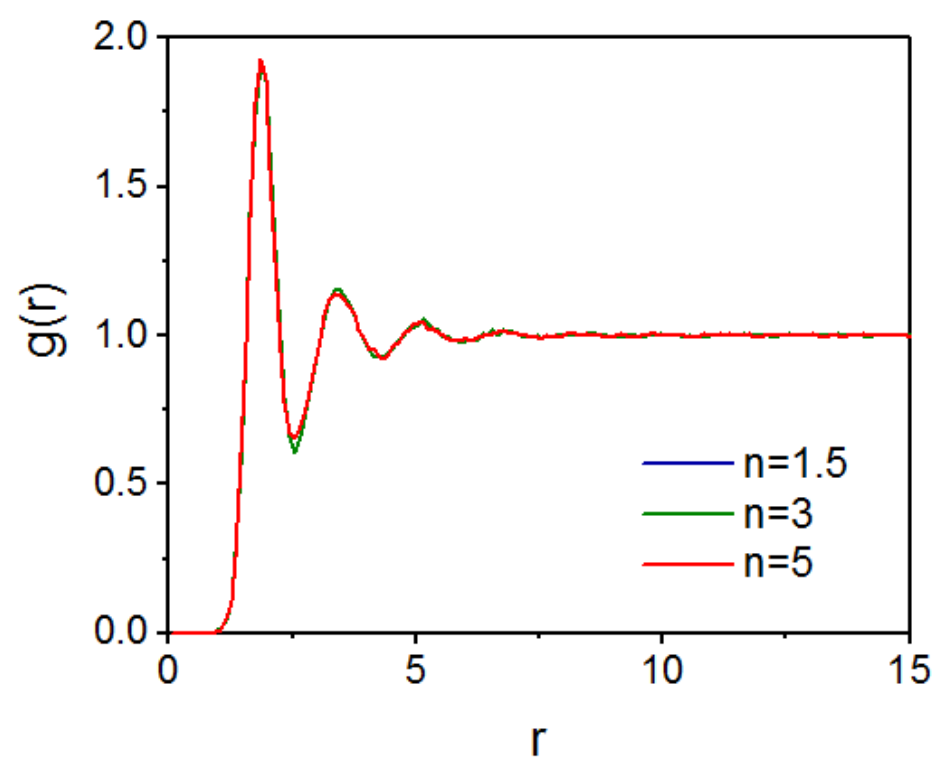

Figure S1 Pairwise particle distribution at rest for volume fraction $=0.8$

2. Fitting parameters for curves in Figs. 2-6. Tables S1-S6 list the fitting parameters used for the curves in Figs. 2-6 of the paper. Standard deviations are given inside parentheses following each data point. All the data shown in Table S1 and S5 are based on the following dimensionless equations:

$$
\begin{gathered}
\sigma / E^{*}=\sigma_{y} / E^{*}+k_{\sigma}\left(\dot{\eta}_{s} / E^{*}\right)^{m}, \\
N_{1} / E^{*}=N_{1 y} / E^{*}+k_{N 1}\left(\dot{\eta} \eta_{s} / E^{*}\right)^{m_{1}}, \\
-N_{2} / E^{*}=-N_{2 y} / E^{*}+k_{N 2}\left(\dot{\gamma}_{s} / E^{*}\right)^{m_{2}} .
\end{gathered}
$$


Table S1 Fitting parameters for curves in Fig. 2.

\begin{tabular}{|c|c|c|c|}
\hline Volume Fraction & $\sigma_{y}$ & $k_{\sigma}$ & $m$ \\
\hline 0.70 & $1.36 \times 10^{-4}\left(1.59 \times 10^{-5}\right)$ & $2.93(2.52)$ & $0.606(0.065)$ \\
\hline 0.80 & $6.29 \times 10^{-4}\left(4.59 \times 10^{-5}\right)$ & $1.86(0.79)$ & $0.500(0.032)$ \\
\hline 0.90 & $2.69 \times 10^{-3}\left(9.61 \times 10^{-5}\right)$ & $3.51(0.71)$ & $0.488(0.015)$ \\
\hline \hline Volume Fraction & $N_{1 y}$ & $k_{N 1}$ & $m_{1}$ \\
\hline 0.70 & $1.15 \times 10^{-5}\left(2.00 \times 10^{-6}\right)$ & $0.838(0.354)$ & $0.693(0.036)$ \\
\hline 0.80 & $6.76 \times 10^{-5}\left(1.29 \times 10^{-5}\right)$ & $0.301(0.186)$ & $0.535(0.053)$ \\
\hline 0.90 & $2.49 \times 10^{-4}\left(2.14 \times 10^{-5}\right)$ & $0.666(0.117)$ & $0.538(0.017)$ \\
\hline \hline Volume Fraction & $N_{2 y}$ & $k_{N 2}$ & $m_{2}$ \\
\hline 0.70 & $2.49 \times 10^{-5}\left(1.18 \times 10^{-6}\right)$ & $1.98(0.33)$ & $0.669(0.013)$ \\
\hline 0.80 & $1.11 \times 10^{-4}\left(6.59 \times 10^{-6}\right)$ & $2.02(0.24)$ & $0.615(0.011)$ \\
\hline 0.90 & $3.48 \times 10^{-4}\left(3.06 \times 10^{-5}\right)$ & $2.77(0.39)$ & $0.587(0.013)$ \\
\hline
\end{tabular}

(The fitting parameters are similar for simulations with and without near-field draft forces.)

Table S2 Parameters for static properties in Figs. 3 and 4.

\begin{tabular}{|c|c|c|c|c|c|c|}
\hline \multirow{2}{*}{$n$} & \multirow{2}{*}{ Parameters } & \multicolumn{5}{|c|}{ Volume fraction } \\
\cline { 2 - 7 } & & 0.70 & 0.75 & 0.80 & 0.85 & 0.90 \\
\hline \hline \multirow{4}{*}{1.5} & $\varepsilon$ & 0.079 & 0.140 & 0.196 & 0.251 & 0.300 \\
\cline { 2 - 7 } & $Z$ & 8.04 & 8.86 & 9.51 & 10.04 & 10.51 \\
\cline { 2 - 7 } & $G$ & $9.22 \times 10^{-3}$ & $1.85 \times 10^{-2}$ & $2.79 \times 10^{-2}$ & $3.71 \times 10^{-2}$ & $4.72 \times 10^{-2}$ \\
& & $(0.00149)$ & $(0.00258)$ & $(0.00224)$ & $(0.00403)$ & $(0.00356)$ \\
\hline \multirow{4}{*}{3} & $\varepsilon$ & 0.091 & 0.159 & 0.219 & 0.276 & 0.329 \\
\cline { 2 - 7 } & $Z$ & 7.85 & 8.57 & 9.13 & 9.62 & 10.04 \\
\cline { 2 - 7 } & $G$ & $3.63 \times 10^{-4}$ & $1.82 \times 10^{-3}$ & $4.95 \times 10^{-3}$ & $1.00 \times 10^{-2}$ & $1.56 \times 10^{-2}$ \\
\hline \multirow{4}{*}{5} & $\left(6.09 \times 10^{-5}\right)$ & $\left(2.87 \times 10^{-4}\right)$ & $\left(4.41 \times 10^{-4}\right)$ & $\left(1.13 \times 10^{-3}\right)$ & $\left(2.23 \times 10^{-3}\right)$ \\
\cline { 2 - 7 } & & 0.099 & 0.169 & 0.232 & 0.292 & 0.346 \\
\cline { 2 - 7 } & $G$ & 7.73 & 8.41 & 8.94 & 9.38 & 9.75 \\
\hline
\end{tabular}

Table S3 Fitting parameters for low frequency modulus in Fig. 3.

\begin{tabular}{|c|c|c|c|}
\hline $\boldsymbol{n}$ & $G_{0}$ & $\phi_{c}$ & $\gamma$ (fixed to $n$-0.5) \\
\hline $\mathbf{1 . 5}$ & $0.188(0.001)$ & $0.651(0.001)$ & 1.0 \\
\hline $\mathbf{3}$ & $0.508(0.021)$ & $0.645(0.001)$ & 2.5 \\
\hline $\mathbf{5}$ & $1.460(0.090)$ & $0.641(0.002)$ & 4.5 \\
\hline
\end{tabular}

Note: $G=G_{0}\left(\phi-\phi_{c}\right)^{\gamma}$

Table S4 Fitting parameters in Fig. 4

\begin{tabular}{|c|c|c|c||c|c|}
\hline \multirow{2}{*}{$\boldsymbol{n}$} & \multicolumn{3}{|c||}{ Fig. 4a } & \multicolumn{2}{c|}{ Fig. 4b } \\
\cline { 2 - 6 } & $Z_{c}$ & $Z_{0}$ & $\zeta$ & $\mathcal{E}_{0}$ & $\phi_{c}$ \\
\hline $\mathbf{1 . 5}$ & $6.0(0.1)$ & $8.96(0.03)$ & $0.49(0.02)$ & $1.24(0.03)$ & $0.63(0.01)$ \\
\hline $\mathbf{3}$ & $6.3(0.1)$ & $7.98(0.04)$ & $0.54(0.02)$ & $1.19(0.03)$ & $0.62(0.01)$ \\
\hline $\mathbf{5}$ & $6.0(0.1)$ & $7.32(0.04)$ & $0.49(0.02)$ & $1.10(0.02)$ & $0.62(0.01)$ \\
\hline
\end{tabular}

Note: $Z-Z_{c}=Z_{0}\left(\phi-\phi_{c}\right)^{\zeta}$ with $\phi_{c}$ fixed to $0.65 ; \varepsilon=\varepsilon_{0}\left(\phi-\phi_{c}\right)$ with $\phi_{c}$ not fixed. 
Table S5.1 Fitting parameters for shear stress in Fig. 5.

\begin{tabular}{|c|c|c|c|c|}
\hline \multirow{3}{*}{$\boldsymbol{n}$} & Volume Fraction & $\sigma_{y}$ & $k_{\sigma}$ & $m$ \\
\hline \hline \multirow{4}{*}{1.5} & 0.70 & $2.01 \times 10^{-4}\left(1.6 \times 10^{-6}\right)$ & $0.517(0.042)$ & $0.438(0.005)$ \\
\cline { 2 - 5 } & 0.75 & $5.69 \times 10^{-4}\left(6.0 \times 10^{-6}\right)$ & $0.499(0.055)$ & $0.413(0.008)$ \\
\cline { 2 - 5 } & 0.80 & $1.07 \times 10^{-3}\left(7.8 \times 10^{-6}\right)$ & $0.637(0.097)$ & $0.415(0.011)$ \\
\cline { 2 - 5 } & 0.85 & $1.66 \times 10^{-3}\left(3.5 \times 10^{-5}\right)$ & $0.571(0.102)$ & $0.398(0.014)$ \\
\cline { 2 - 5 } & 0.90 & $1.83 \times 10^{-3}\left(4.5 \times 10^{-5}\right)$ & $0.344(0.086)$ & $0.341(0.018)$ \\
\hline \hline \multirow{4}{*}{3} & 0.70 & $6.71 \times 10^{-6}\left(3.7 \times 10^{-7}\right)$ & $0.475(0.073)$ & $0.517(0.009)$ \\
\cline { 2 - 5 } & 0.75 & $4.51 \times 10^{-5}\left(7.8 \times 10^{-7}\right)$ & $0.349(0.031)$ & $0.461(0.006)$ \\
\cline { 2 - 5 } & 0.80 & $1.39 \times 10^{-4}\left(7.0 \times 10^{-7}\right)$ & $0.404(0.017)$ & $0.441(0.003)$ \\
\cline { 2 - 5 } & 0.85 & $3.00 \times 10^{-4}\left(1.7 \times 10^{-6}\right)$ & $0.436(0.016)$ & $0.424(0.003)$ \\
\hline \hline \multirow{3}{*}{5} & 0.90 & $5.56 \times 10^{-4}\left(7.5 \times 10^{-6}\right)$ & $0.504(0.046)$ & $0.417(0.006)$ \\
\cline { 2 - 5 } & 0.70 & $1.00 \times 10^{-7}\left(1.5 \times 10^{-8}\right)$ & $1.015(0.15)$ & $0.637(0.007)$ \\
\cline { 2 - 5 } & 0.75 & $1.84 \times 10^{-6}\left(2.0 \times 10^{-7}\right)$ & $0.469(0.087)$ & $0.551(0.011)$ \\
\cline { 2 - 5 } & 0.80 & $1.06 \times 10^{-5}\left(2.2 \times 10^{-7}\right)$ & $0.350(0.054)$ & $0.500(0.009)$ \\
\cline { 2 - 5 } & 0.85 & $3.65 \times 10^{-5}\left(5.3 \times 10^{-7}\right)$ & $0.394(0.051)$ & $0.476(0.008)$ \\
\hline
\end{tabular}

Table S5.2 Fitting parameters for first normal stress difference $N_{1}$ in Fig. 5.

\begin{tabular}{|c|c|c|c|c|}
\hline $\boldsymbol{n}$ & Volume Fraction & $N_{1 y}$ & $k_{N 1}$ & $m_{1}$ \\
\hline \multirow{4}{*}{1.5} & 0.70 & $1.91 \times 10^{-5}\left(2.0 \times 10^{-6}\right)$ & $0.650(0.335)$ & $0.629(0.041)$ \\
\cline { 2 - 5 } & 0.75 & $5.70 \times 10^{-5}\left(2.0 \times 10^{-6}\right)$ & $0.424(0.061)$ & $0.554(0.012)$ \\
\cline { 2 - 5 } & 0.80 & $1.07 \times 10^{-4}\left(3.5 \times 10^{-6}\right)$ & $0.456(0.059)$ & $0.529(0.011)$ \\
\cline { 2 - 5 } & 0.85 & $1.68 \times 10^{-4}\left(4.3 \times 10^{-6}\right)$ & $0.487(0.061)$ & $0.511(0.010)$ \\
\cline { 2 - 5 } & 0.90 & $2.10 \times 10^{-4}\left(1.4 \times 10^{-5}\right)$ & $0.359(0.102)$ & $0.464(0.024)$ \\
\hline \hline \multirow{4}{*}{3} & 0.70 & $5.95 \times 10^{-7}\left(9.9 \times 10^{-8}\right)$ & $0.232(0.101)$ & $0.645(0.029)$ \\
\cline { 2 - 5 } & 0.75 & $4.42 \times 10^{-6}\left(3.2 \times 10^{-7}\right)$ & $0.260(0.091)$ & $0.607(0.025)$ \\
\cline { 2 - 5 } & 0.80 & $1.39 \times 10^{-5}\left(1.7 \times 10^{-6}\right)$ & $0.402(0.132)$ & $0.596(0.026)$ \\
\cline { 2 - 5 } & 0.85 & $3.26 \times 10^{-5}\left(1.6 \times 10^{-6}\right)$ & $0.419(0.065)$ & $0.572(0.013)$ \\
\hline \hline \multirow{4}{*}{5} & 0.90 & $5.70 \times 10^{-5}\left(1.9 \times 10^{-6}\right)$ & $0.342(0.048)$ & $0.534(0.011)$ \\
\cline { 2 - 5 } & 0.70 & $1.45 \times 10^{-8}\left(2.1 \times 10^{-9}\right)$ & $0.147(0.072)$ & $0.707(0.027)$ \\
\cline { 2 - 5 } & 0.75 & $1.93 \times 10^{-7}\left(5.2 \times 10^{-8}\right)$ & $0.304(0.245)$ & $0.694(0.051)$ \\
\cline { 2 - 5 } & 0.80 & $1.08 \times 10^{-6}\left(2.5 \times 10^{-7}\right)$ & $0.113(0.072)$ & $0.589(0.042)$ \\
\cline { 2 - 5 } & 0.85 & $3.60 \times 10^{-6}\left(3.1 \times 10^{-7}\right)$ & $0.090(0.029)$ & $0.537(0.022)$ \\
\hline
\end{tabular}


Table S5.3 Fitting parameters for second normal stress difference $N_{2}$ in Fig. 5 .

\begin{tabular}{|c|c|c|c|c|}
\hline \multirow{n}{*}{$\mathbf{n}$} & Volume Fraction & $-N_{2 y}$ & $k_{N 2}$ & $m_{2}$ \\
\hline \hline \multirow{4}{*}{1.5} & 0.70 & $2.88 \times 10^{-5}\left(1.1 \times 10^{-6}\right)$ & $0.487(0.042)$ & $0.533(0.007)$ \\
\cline { 2 - 5 } & 0.75 & $7.40 \times 10^{-5}\left(9.1 \times 10^{-7}\right)$ & $0.421(0.014)$ & $0.501(0.003)$ \\
\cline { 2 - 5 } & 0.80 & $1.28 \times 10^{-4}\left(2.0 \times 10^{-6}\right)$ & $0.466(0.020)$ & $0.496(0.004)$ \\
\cline { 2 - 5 } & 0.85 & $1.79 \times 10^{-4}\left(4.9 \times 10^{-6}\right)$ & $0.481(0.051)$ & $0.487(0.009)$ \\
\cline { 2 - 5 } & 0.90 & $2.19 \times 10^{-4}\left(8.6 \times 10^{-6}\right)$ & $0.422(0.054)$ & $0.469(0.011)$ \\
\hline \hline \multirow{4}{*}{3} & 0.70 & $9.35 \times 10^{-7}\left(1.8 \times 10^{-7}\right)$ & $0.661(0.121)$ & $0.621(0.012)$ \\
\cline { 2 - 5 } & 0.75 & $6.68 \times 10^{-6}\left(5.6 \times 10^{-7}\right)$ & $0.577(0.118)$ & $0.584(0.014)$ \\
\cline { 2 - 5 } & 0.80 & $1.90 \times 10^{-5}\left(1.1 \times 10^{-6}\right)$ & $0.508(0.038)$ & $0.551(0.006)$ \\
\cline { 2 - 5 } & 0.85 & $3.96 \times 10^{-5}\left(1.5 \times 10^{-6}\right)$ & $0.557(0.048)$ & $0.539(0.007)$ \\
\hline \hline \multirow{4}{*}{5} & 0.90 & $6.70 \times 10^{-5}\left(1.2 \times 10^{-6}\right)$ & $0.527(0.022)$ & $0.518(0.004)$ \\
\cline { 2 - 5 } & 0.70 & $1.60 \times 10^{-8}\left(3.0 \times 10^{-9}\right)$ & $0.990(0.093)$ & $0.710(0.005)$ \\
\cline { 2 - 5 } & 0.75 & $3.55 \times 10^{-7}\left(5.6 \times 10^{-8}\right)$ & $0.821(0.125)$ & $0.664(0.010)$ \\
\cline { 2 - 5 } & 0.80 & $1.77 \times 10^{-6}\left(2.8 \times 10^{-7}\right)$ & $0.635(0.139)$ & $0.620(0.015)$ \\
\cline { 2 - 5 } & 0.85 & $5.08 \times 10^{-6}\left(5.6 \times 10^{-7}\right)$ & $0.511(0.103)$ & $0.580(0.014)$ \\
\hline
\end{tabular}

Table S6. Fitting parameters in Fig. 6.

\begin{tabular}{|c|c|c|c|}
\hline $\mathbf{n}$ & & $\tilde{k}_{\sigma}$ & $m$ \\
\cline { 1 - 2 } $\mathbf{1 . 5}$ & & $145.7(37.2)$ & $0.407(0.025)$ \\
\cline { 3 - 4 } $\mathbf{3}$ & & $224.2(20.4)$ & $0.428(0.009)$ \\
\cline { 3 - 4 } $\mathbf{5}$ & & $677.0(73.5)$ & $0.503(0.012)$ \\
\hline \hline $\mathbf{n}$ & $\tilde{N}_{1 y}$ & $\tilde{k}_{N 1}$ & $\tilde{m}_{1}$ \\
\hline $\mathbf{1 . 5}$ & $0.104(0.007)$ & $72.5(15.2)$ & $0.537(0.025)$ \\
\hline $\mathbf{3}$ & $0.104(0.005)$ & $112.4(12.0)$ & $0.588(0.014)$ \\
\hline $\mathbf{5}$ & $0.110(0.007)$ & $132.2(15.5)$ & $0.616(0.016)$ \\
\hline $\mathbf{n}$ & $-\tilde{N}_{2 y}$ & $\tilde{k}_{N 2}$ & $\tilde{m}_{2}$ \\
\hline $\mathbf{1 . 5}$ & $0.133(0.023)$ & $112.9(49.8)$ & $0.532(0.053)$ \\
\hline $\mathbf{3}$ & $0.136(0.027)$ & $181.0(45.6)$ & $0.550(0.033)$ \\
\hline $\mathbf{5}$ & $0.144(0.026)$ & $420.2(75.2)$ & $0.611(0.023)$ \\
\hline
\end{tabular}

Note: $\sigma / \sigma_{y}=1+\tilde{k}_{\sigma}\left(\dot{\eta} \eta_{s} / G_{0}\right)^{m}$

$N_{1} / \sigma_{y}=\tilde{N}_{1 y}+\tilde{k}_{N 1}\left(\dot{\eta} \eta_{s} / G_{0}\right)^{\tilde{m}_{1}} ;-N_{2} / \sigma_{y}=-\tilde{N}_{2 y}+\tilde{k}_{N 2}\left(\dot{\gamma} \eta_{s} / G_{0}\right)^{\tilde{m}_{2}}$

Table S7. Fitting parameters in Fig 7.

\begin{tabular}{|c|c|c|}
\hline & $\bar{k}_{\sigma}$ & $\bar{m}$ \\
\cline { 2 - 3 } & $42.6(7.9)$ & $0.405(0.025)$ \\
\hline \hline $\bar{N}_{1 y}$ & $\bar{k}_{N 1}$ & $\bar{m}_{1}$ \\
\hline $0.104(0.005)$ & $14.7(1.4)$ & $0.540(0.018)$ \\
\hline$-\bar{N}_{2 y}$ & $\bar{k}_{N 2}$ & $\bar{m}_{2}$ \\
\hline $0.130(0.026)$ & $20.1(6.3)$ & $0.517(0.058)$ \\
\hline
\end{tabular}

(Note: $\sigma / \sigma_{y}=1+\bar{k}_{\sigma}\left(\dot{\gamma} \eta_{s} / \gamma_{y}^{2} E^{*}\right)^{\bar{m}}$

$\left.N_{1} / \sigma_{y}=\bar{N}_{1 y}+\bar{k}_{N 1}\left(\dot{\eta} \eta_{s} / \gamma_{y}^{2} E^{*}\right)^{\bar{m}_{1}} ;-N_{2} / \sigma_{y}=-\bar{N}_{2 y}+\bar{k}_{N 2}\left(\dot{\eta} \eta_{s} / \gamma_{y}^{2} E^{*}\right)^{\bar{m}_{2}}\right)$ 
3. Scaling of yield stress with distance to jamming.
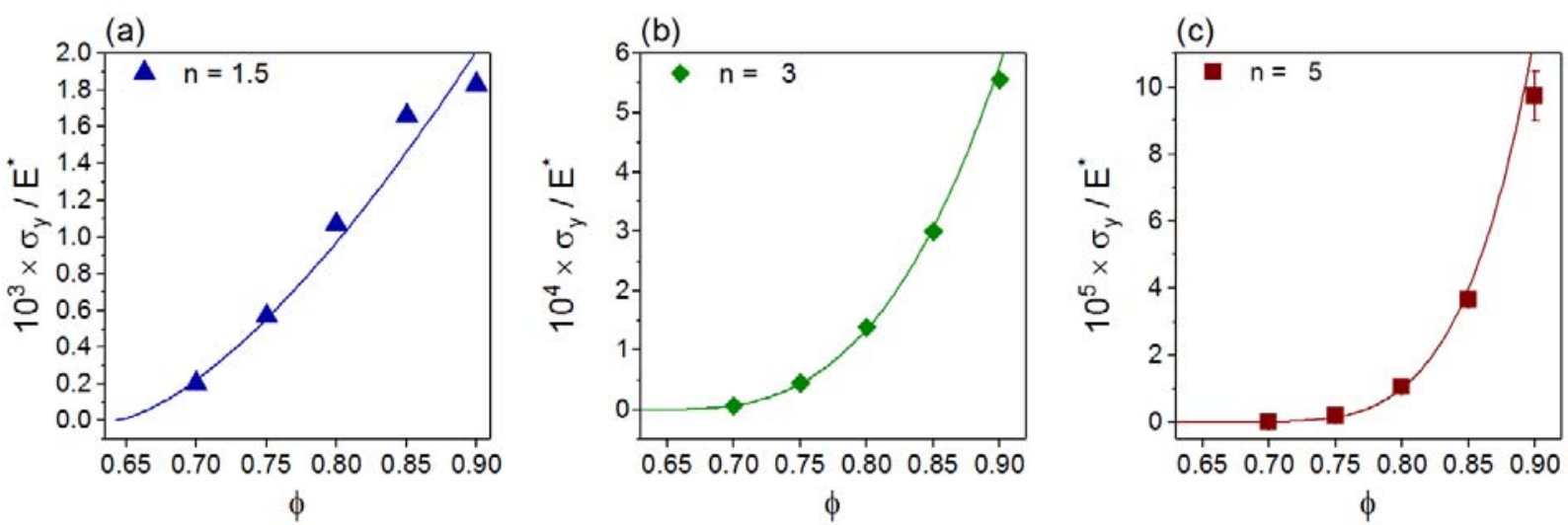

Figure S2 Scaling of yield stress with volume fraction. $\phi_{c}$ is assumed to be 0.64 . The fitted curves are:

(a) $\sigma_{y} / E^{*}=0.0152\left(\phi-\phi_{c}\right)^{1.5}$; (b) $\sigma_{y} / E^{*}=0.0331\left(\phi-\phi_{c}\right)^{3}$; (c) $\sigma_{y} / E^{*}=0.0980\left(\phi-\phi_{c}\right)^{5}$. 